Bundesgesundheitsbl 2016 $\cdot 59: 1606-1622$ DOI 10.1007/s00103-016-2467-8

Online publiziert: 4. November 2016

(c) Springer-Verlag Berlin Heidelberg 2016

Cornelius Remschmidt ${ }^{1}$. Thomas Harder ${ }^{1}$. Ole Wichmann ${ }^{1}$ - Edeltraut Garbe ${ }^{2}$. Thomas Ledig ${ }^{3} \cdot$ Martin Terhardt $^{4} \cdot$ Sabine Wicker $^{5} \cdot$ Fred Zepp $^{6} \cdot$ Thomas Mertens $^{7}$

1 Fachgebiet Impfprävention, Robert Koch-Institut, Berlin, Deutschland

${ }^{2}$ Klinische Epidemiologie, Leibniz-Institut für Präventionsforschung und Epidemiologie - BIPS GmbH, Bremen, Deutschland

${ }^{3}$ Abteilung Allgemeinmedizin und Versorgungsforschung, Universitätsklinikum Heidelberg, Heidelberg, Deutschland

${ }^{4}$ Medizinisches Versorgungszentrum am Hermannplatz, Berlin, Deutschland

${ }^{5}$ Betriebsärztlicher Dienst, Universitätsklinikum Frankfurt, Frankfurt am Main, Deutschland

${ }^{6}$ Zentrum für Kinder- und Jugendmedizin, Universitätsmedizin Mainz, Mainz, Deutschland

${ }^{7}$ Institut für Virologie, Universitätsklinikum Ulm, Ulm, Deutschland

\title{
Hintergrundpapier der STIKO: Evaluation der bestehenden Influenzaimpfempfehlung für Indikationsgruppen und für Senioren (Standardimpfung ab 60 Jahren)
}

\section{Zusatzmaterial online \\ In der Online-Version dieses Artikels (doi:10.1007/s00103-016-2467-8) finden Sie den Anhang zum Hintergrundpapier der STIKO.}

\section{Hintergrund}

\section{Influenzaviren}

Influenzaviren gehören zu den Orthomyxoviren und werden in die Typen A, $B$ und $C$ eingeteilt, wobei für Menschen nur die Influenza-A- und -B-Viren medizinisch relevant sind. Eine Einteilung in weitere Subtypen erfolgt bei den Influenza-B-Viren in zwei genetisch unterschiedliche Linien (die Victoria- und die Yamagata-Linie) und bei den InfluenzaA-Viren anhand der Oberflächenproteine Hämagglutinin (HA) und Neuraminidase (NA), von denen mittlerweile 18 HA- und 11 NA-Typen identifiziert wurden [1]. In den vergangenen Jahrzehnten zirkulierten v. a. die Influenza-A-Viren $\mathrm{H} 3 \mathrm{~N} 2$ und $\mathrm{H} 1 \mathrm{~N} 1$ sowie Influenza-B-Vi- ren der Victoria- und die Yamagata-Linie [2]. Die genaue (englische) Bezeichnung der Influenzaviren setzt sich zusammen aus dem Influenzatyp (A oder B), dem ursprünglichen Wirt des Subtypen (z. B. swine, chicken; bei humanem Ursprung wird diese Namenskomponente weggelassen), dem Ort der ersten Isolierung (z. B. California, Brisbane), einer Nummer des Isolats, dem Jahr der Isolation (z.B. 57 für 1957 oder 2009) und bei Influenza-A-Viren die Bezeichnung des HA- und des NA-Typs (z.B. H1N1 oder H3N2) [3]. So wurde das während der Pandemie zirkulierende Virus mit der Bezeichnung A/California/7/2009 (H1N1) versehen. Häufig wird nur die Kurzbezeichnung der Influenzaviren angegeben, also Influenza B in Kombination mit der entsprechenden Linie (z.B. Influenza B/Yamagata) bzw. bei den AViren der entsprechende Subtyp (z. B. A/ H3N2).

Eine Besonderheit der Influenzaviren ist ihre große genetische Variabilität. Durch ständig stattfindende Mutationen verändern sich die RNA-Viren kontinu- ierlich, wobei die Mutationsrate beim Influenza-A-Virus höher ist als beim Influenza-B-Virus [4]. Durch Punktmutationen verursachte genetische Veränderungen werden als Antigendrift bezeichnet. Größere, durch Reassortierung bedingte Veränderungen bezeichnet man als Antigenshift, die zur Entstehung neuer pandemischer Influenzaviren führen können. Grundlage der Reassortierung bei Influenza-A-Viren ist auch die Tatsache, dass Influenza-A-Viren viele extrahumane Wirte infizieren können.

Bereits durch Antigendrifts kann sich das Influenzavirus derart verändern, dass Antikörper, die gegen frühere Varianten des Influenzasubtyps gebildet wurden, keinen oder nur einen partiellen Schutz gegen eine Infektion durch die veränderte Variante bieten [5]. Dadurch fehlt nach durchgemachter Infektion oder nach Impfung eine langanhaltende Immunität, was wiederum zum Auftreten jährlicher Influenzawellen führt. Bei einem Antigenshift können größere genetische Veränderungen durch Austausch von Gensegmenten (Reassortierung) zu einer Influenza- 


\section{Zusammenfassung}

\section{Hintergrund und Fragestellung}

Ziel der vorliegenden Untersuchung war es, die lange bestehende Influenzaimpfempfehlung der Ständigen Impfkommission (STIKO) für Indikationsgruppen und für Senioren entsprechend der neuen Standardvorgehensweise für die systematische Entwicklung von Impfempfehlungen nachträglich zu evaluieren. Die Evidenz zur Effektivität und Sicherheit der saisonalen Influenzaimpfung wurde für die Indikationsgruppen und Senioren systematisch untersucht und die Qualität der Evidenz entsprechend der GRADE („Grading of Recommendations Assessment, Development and Evaluation")-Methodik in vier Stufen - sehr niedrige, niedrige, moderate oder hohe Evidenzqualität - eingeteilt. Zusätzlich wurde mittels orientierender Literatursuchen analysiert, inwieweit in den untersuchten Personengruppen bei Influenzainfektionen ein erhöhtes Risiko für schwere Krankheitsverläufe besteht.

Die STIKO kommt auf der Grundlage dieser Daten zu folgender Bewertung:

\section{Effektivität und Sicherheit der Influenzaimpfung in den Indikationsgruppen}

- Für die Gruppe der Schwangeren und deren Neugeborenen liegt Evidenz von hoher Qualität vor, dass die Impfung während der Schwangerschaft vor laborbestätigten Influenzainfektionen schützt. In den analysierten Studienzeiträumen wurde sowohl bei den Schwangeren als auch bei den Neugeborenen eine Impfeffektivität von $50 \%$ gegen laborbestätigte Influenzainfektionen beobachtet.

- Für die Indikationsgruppen der HIV-positiven Erwachsenen liegt Evidenz von moderater Qualität vor, dass die Impfung vor laborbestätigten Influenzainfektionen schützt. Bei HIV-positiven Kleinkindern hingegen fand sich keine Evidenz für einen protektiven Effekt.

- Für Personen mit Herz-Kreislauf-Erkrankungen liegt Evidenz von hoher Qualität vor, dass die Impfung vor schweren kardiovaskulären Ereignissen schützt. Der größte Effekt wurde bei Personen mit einem erst kurz zurückliegenden koronarischämischen Ereignis beobachtet.

- Für medizinisches Personal liegt Evidenz von niedriger bis moderater Qualität vor, dass die Impfung des medizinischen Personals bei den von innen betreuten (älteren) Patienten Todesfälle mit einer Effektivität von 30\% und influenzaähnliche Erkrankungen mit einer Effektivität von $42 \%$ verhindern kann. Eine Wirksamkeit der Impfung gegen laborbestätigte Erkrankungen bei den betreuten Patienten konnte nur in Beobachtungsstudien gezeigt werden, die Evidenzqualität war hier sehr niedrig.

- Für Personen mit Diabetes mellitus bzw. mit terminaler Niereninsuffizienz liegt Evidenz von sehr niedriger bis niedriger Qualität vor, dass die Impfung gegen unspezifische Endpunkte (z. B. Hospitalisierung) schützt. Die beobachtete Impfeffektivität lag je nach Endpunkt und Altersgruppe zwischen $13 \%$ und $58 \%$. Allerdings sind die Effektschätzer mit großer Unsicherheit behaftet, insbesondere, weil Studien mit laborbestätigten Endpunkten fehlen.

- Für Erwachsene mit Asthma bronchiale oder mit chronisch obstruktiver Lungenerkrankung (COPD) konnten keine Studien zur Wirksamkeit der Impfung identifiziert werden. Bei Kindern mit Asthma bronchiale liegt Evidenz von moderater Qualität vor, dass die Impfung keinen protektiven Effekt gegen influenzabedingte Asthmaexazerbationen zeigt.

- In keiner der untersuchten Indikationsgruppen wurden Hinweise auf eine Häufung von schweren unerwünschten Arzneimittelwirkungen nach saisonaler Influenzaimpfung gefunden.

Effektivität und Sicherheit der Influenzaimpfung bei Senioren

- Für Senioren in Pflegeinstitutionen liegt Evidenz von moderater, für selbstständig lebende („„community dwelling") Senioren Evidenz von hoher Qualität vor, dass die Impfung vor laborbestätigten Influenzainfektionen schützt. Die in randomisierten kontrollierten Studien (RCTs) gemessene Impfeffektivität von $50 \%$ konnte in Beobachtungsstudien mit niedrigem Verzerrungspotenzial bestätigt werden.

- In der Literatur konnten auch hier keine Hinweise auf eine Häufung von schweren unerwünschten Arzneimittelwirkungen nach saisonaler Influenzaimpfung gefunden werden.

Risiko für schwere Krankheitsverläufe in den Indikationsgruppen und bei Senioren bei Influenzainfektionen

- In der Literatur zeigte sich, dass bei Personen mit einer Grunderkrankung im Sinne der STIKO-Indikationsgruppen, bei Schwangeren und bei Senioren ein erhöhtes Risiko für schwere Krankheitsverläufe besteht, z. B. gemessen als Hospitalisierung, Entwicklung einer Pneumonie oder Einlieferung auf eine Intensivstation.

Schlussfolgerung

- Ziel der Impfung gegen Influenza ist weiterhin die Reduktion von Influenzaerkrankungen in besonders gefährdeten Risikogruppen und die hieraus resultierenden Folgen wie Hospitalisierung und Tod.

- Angesichts der hohen Krankheitslast, die durch die jährlichen Influenzawellen verursacht wird, stellt die Influenzaimpfung auch bei vergleichsweise geringer Impfeffektivität unverändert eine sinnvolle Präventionsmaßnahme dar, da selbst bei einer moderaten Impfeffektivität viele Influenzaerkrankungen verhindert werden können.

- Auf Grundlage der vorliegenden Evidenz belässt die STIKO ihre Impfempfehlung für die genannten Indikationsgruppen und für Senioren, zumal bei diesen ein erhöhtes Risiko für schwere Krankheitsverläufe besteht. Die STIKO weist darauf hin, dass die evaluierten Indikationsgruppen exemplarischen Charakter haben und keine abschließende Indikationsliste darstellen.

- Angesichts der Daten zur Wirksamkeit und Sicherheit der Impfung von Schwangeren und von medizinischem Personal sieht die STIKO eine hohe Bedeutung der Impfung dieser Risikogruppen zum Schutz Dritter (Neugeborene, betreute Patienten).

- Bei der Bewertung der gefundenen Evidenz muss bedacht werden, dass eine Berechnung der Impfeffektivität immer retrospektiv erfolgt und eine Prognose für zukünftige Influenzasaisons nicht möglich ist, weil die Effektivität stark vom jeweils kursierenden Virus abhängt. Die STIKO ist sich bewusst, dass viele der identifizierten Studien nicht in Deutschland durchgeführt wurden und eine Übertragbarkeit der Daten auf die hiesige Situation je nach untersuchtem Parameter eingeschränkt sein könnte.

- Die STIKO betont die Notwendigkeit der Durchführung weiterer Studien mit aussagekräftigem Studiendesign und laborbestätigten Endpunkten, um die Qualität der Evidenz zur saisonalen Influenzaimpfung zu verbessern und bestehende Evidenzlücken zu schließen.

- Die Forschung zur Entwicklung neuer, besserer und breiter wirksamer Impfstoffe muss vorangetrieben werden. 
variante führen, die in dieser Form bisher nicht in der menschlichen Bevölkerung zirkulierte (z.B. A(H2N2) im Jahr 1957) bzw. deren neuer Subtyp sich erheblich von den bisher zirkulierenden Subtypen unterscheidet (z.B. das A(H1N1)pdm09 im Jahr 2009).

\section{Klinik und Diagnostik der Influenza}

Die influenzatypische Erkrankung (,,influenza-like illness", ILI) ist gekennzeichnet durch plötzlichen Erkrankungsbeginn mit hohem Fieber, trockenem Husten und Hals-, Muskel- und/oder Gliederschmerzen [6]. In Abhängigkeit vom Virus bzw. einer vorbestehenden Immunität erkrankt allerdings häufig ein großer Teil der Infizierten mit milderen Symptomen bzw. weist einen asymptomatischen Verlauf auf [7, 8]. Die Krankheitsdauer liegt durchschnittlich bei 5-7 Tagen; allerdings kann die Krankheit, insbesondere beim Vorliegen von bestimmten Risikofaktoren, länger andauern. Bei schweren Krankheitsverläufen stehen pulmonale Komplikationen wie Pneumonien im Vordergrund. Weitere mögliche Folgen einer schweren Influenzaerkrankung sind Myositiden oder eine kardiale bzw. zerebrale Symptomatik [6]. Bei Kindern zählt die Otitis media zu den die häufigsten Komplikationen. Ein erhöhtes Risiko für Komplikationen haben Personen mit chronischen Vorerkrankungen und Kleinkinder $[9,10]$.

Die Diagnose der Influenza ist anhand der klinischen Symptomatik (ILI) nicht sicher möglich [11]. Die Verlässlichkeit der klinischen Diagnose nimmt jedoch während einer Influenzawelle deutlich zu [12]. Mittels Virusisolierung bzw. durch den Nachweis von Virus-RNA mittels rtPCR (reverse Transkriptase-Polymerase-Kettenreaktion) kann die Diagnose mit hoher Sicherheit gestellt werden [6], wobei sich die PCR (Polymerase-Kettenreaktion) hier als labordiagnostischer Goldstandard etabliert hat.

\section{Epidemiologie und Krankheitslast der Influenza}

Influenzaviren zirkulieren weltweit und verursachen in den gemäßigten Zonen der Erde jährlich Grippewellen, die auf der Südhalbkugel während der Monate April bis Juli und auf der nördlichen Halbkugel in den Wintermonaten auftreten. Die Weltgesundheitsorganisation (WHO) schätzt, dass während der jährlichen Influenzawellen durchschnittlich $20-30 \%$ der Kinder und 5-10\% der Erwachsenen von einer Influenzavirusinfektion betroffen sind und jährlich ca. 250.000-500.000 Personen an den Folgen einer Influenzaerkrankung sterben [13]. In Deutschland verursachen die saisonalen Influenzawellen jährlich schätzungsweise bis zu 7 Mio. Arztkonsultationen und bis zu 32.000 Hospitalisationen [14, 15].

\section{Saisonale Influenzaimpfstoffe}

In Deutschland stehen für die saisonale Influenzaimpfung inaktivierte, lebendattenuierte und inaktivierte-adjuvantierte Impfstoffe zur Verfügung [16, 17]. Die Impfstoffe enthalten antigene Bestandteile derjenigen Influenzasubtypen, die von der WHO im Rahmen der jährlichen Impfstoffanpassung empfohlen werden (http://www.who.int/influenza/vaccines/ virus/recommendations/en/). Bis zum Jahr 2011 waren in den Impfstoffen Antigene von zwei Influenza-A-Subtypen und einer Influenza-B-Linie enthalten (trivalenter Impfstoff; ,trivalent influenza vaccine“ - TIV), seit 2012 sind auch Impfstoffe mit Antigenen von vier Influenzaviren (jeweils zwei Influenza-A-Subtypen und beide B-Linien) zugelassen (quadrivalenter Impfstoff, QIV).

\section{Impfeffektivität saisonaler Influenzaimpfstoffe}

Die klinische Effektivität („vaccine efficacy“, VE) von Influenzaimpfstoffen wird idealerweise in randomisierten kontrollierten Studien (RCTs) gemessen. Dabei wird erfasst, wie häufig eine Influenzainfektion bei geimpften im Vergleich zu nichtgeimpften Personen auftritt. Aus dem Quotient dieser Raten, dem relativen Risiko (RR), erfolgt nach der Formel 1 RR die Berechnung der Impfeffektivität, die Angabe erfolgt in Prozent [18]. Da die Durchführung von RCTs personal- und kostenintensiv ist, erfolgt die Bestimmung der Influenzaimpfeffektivität zunehmend in Beobachtungsstudien. Hierbei hat sich gezeigt, dass die Anwendung von Studien mit dem sog. Test-negativen Design (TNDesign) eine valide Alternative zu RCTs darstellt [19]. In Studien mit dem TNDesign wird bei Personen, die sich mit einer akuten Atemwegsinfektion medizinisch vorstellen, eine Probe aus dem Respirationstrakt auf Influenzaviren untersucht. Personen mit Influenzanachweis werden hierbei als „Fälle“, Personen ohne Nachweise des Influenzavirus (negatives PCR-Ergebnis) als „Kontrollen“ bezeichnet. Studien mit dem TN-Design gelten als weniger anfällig für Verzerrungen als Studien, die indirekte (nichtlaborbestätigte) Endpunkte wie ILI oder Hospitalisierungen verwenden [20].

Bei der Beurteilung der VE ist generell $\mathrm{zu}$ berücksichtigen, dass aufgrund starker saisonaler Schwankungen eine Prognose der VE für zukünftige Saisons nicht möglich ist. Diese Schwankungen werden durch eine Vielzahl von häufig nicht vorhersehbaren Faktoren beeinflusst. Hierzu gehört beispielsweise die hohe Variabilität der Influenzaviren, die sich durch fortlaufende Veränderungen genetisch vom Impfvirus entfernen, wodurch sich die Impfeffektivität verringern kann. Aber auch das Alter des Impflings, frühere Influenzainfektionen oder -impfungen und auch die Art des verwendeten Impfstoffs können die Effektivität der Impfung beeinflussen [21-23]. Und letztlich kann sich die Impfeffektivität gegenüber den verschiedenen Influenzatypen und -subtypen deutlich unterscheiden.

\section{Influenzaimpfempfehlung der STIKO}

Die STIKO empfiehlt die jährliche Impfung gegen die saisonale Influenza für alle Personen ab einem Alter von 60 Jahren, für Personen jeden Alters mit erhöhter gesundheitlicher Gefährdung infolge eines Grundleidens (wie z.B. chronische Krankheiten der Atmungsorgane, Herz- oder Kreislaufkrankheiten, Leber- oder Nierenkrankheiten, Diabetes mellitus oder andere Stoffwechselkrankheiten, chronische neurologische Grundkrankheiten, wie z. B. Multiple Sklerose mit durch Infektionen getriggerten Schüben, angeborene oder erworbene Immundefizienz oder HIV-Infektion) sowie für Bewohner von Alters- oder 
Pflegeheimen. Zusätzlich wird die Impfung gesunden Schwangeren ab dem 2. Trimenon und Schwangeren bei erhöhter gesundheitlicher Gefährdung infolge eines Grundleidens ab dem 1. Trimenon empfohlen. Außerdem sollten Personen mit erhöhter beruflicher Gefährdung (z.B. medizinisches Personal) und Personen, die als mögliche Infektionsquelle für von ihnen betreute Risikopersonen fungieren können, gegen saisonale Influenza geimpft werden [24].

\section{Ziel des Hintergrundpapiers}

Der Nutzen der saisonalen Influenzaimpfung wird - insbesondere im höheren Lebensalter oder bei einzelnen Risikogruppen - häufig infrage gestellt. Die STIKO hat daher die bestehende Influenzaimpfempfehlung unter Anwendung der neuen Standardvorgehensweise (SOP) [25] einer Evaluation unterzogen.

Folgende Ziele wurden hierbei verfolgt:

1. Evaluation der Evidenz zur Effektivität und Sicherheit der Influenzaimpfung in verschiedenen Indikationsgruppen und bei Senioren ab einem Alter von 60 Jahren.

2. Erhebung von Daten zum Risiko schwerer Krankheitsverläufe bei Influenzaerkrankungen in den verschiedenen Indikationsgruppen und bei Senioren.

Anhand der erhobenen Daten sollte überprüft werden, ob eine Anpassung der Indikationsimpfempfehlung bzw. der Seniorenimpfempfehlung notwendig ist.

\section{Methodik}

\section{Generelles Vorgehen}

Für die Evaluation der Wirksamkeit und Sicherheit der Influenzaimpfung wurde neben der Gruppe der Senioren (ab 60 Jahren) die Evidenz für die folgenden Indikationsgruppen systematisch bewertet:

- HIV-infizierte Personen,

- Personen mit chronischen Krankheiten der Atmungsorgane (z.B. Asthma),

- Personen mit chronischen Herz-

Kreislauf-Erkrankungen (z. B. koronare Herzkrankheit),

- Personen mit Diabetes mellitus,
- Personen mit Nierenerkrankungen (z.B. Personen mit terminaler Niereninsuffizienz),

- medizinisches Personal,

- Schwangere und deren Kinder.

Bei der Identifikation entsprechender Studien wurde zweistufig vorgegangen. $\mathrm{Zu}$ nächst wurde, soweit vorhanden, auf bereits publizierte systematische Reviews von hoher methodischer Qualität zurückgegriffen. Die methodische Qualität der systematischen Reviews wurde dabei mit dem „Assessment of multiple systematic reviews"(AMSTAR)-Instrument [26] bewertet, wobei maximal 11 Punkte erreicht werden können (AMSTAR-Score). Bei Vorliegen von mehreren systematischen Reviews zu einer Personengruppe wurde neben der methodischen Qualität nach AMSTAR auch die Aktualität des Reviews, d.h. bei gleicher methodischer Qualität der aktuellste Review, berücksichtigt.

Wenn keine methodisch hochwertigen systematischen Reviews identifiziert werden konnten, wurden systematische Reviews von den Autoren durchgeführt. Bei der Durchführung von systematischen Reviews wurde den PRISMA-Guidelines [27] gefolgt.

Bezüglich der Krankheitsschwere wurde mittels orientierender Literaturrecherche evaluiert, ob in den Personengruppen ein erhöhtes Risiko für schwere Influenzaerkrankungen besteht. Bei der Evaluation wurden auch Studien über die Pandemie 2009 berücksichtigt, da das A(H1N1) pdm09-Virus seither saisonal auftretende Influenzaerkrankungen verursacht und entsprechend im saisonalen Influenzaimpfstoff berücksichtigt wird.

\section{Definition patientenrelevanter Endpunkte}

Für alle Indikationsgruppen bzw. für die Gruppe der Senioren wurden von der STIKO - entsprechend der GRADE-Methodik - a priori patientenrelevante Endpunkte zur Wirksamkeit und Sicherheit als „kritisch“, „wichtig“ oder „weniger wichtig" für die Entscheidungsfindung definiert. Alle von der STIKO als „kritisch“ oder „wichtig“ definierten Endpunkte gingen in die Bewertung der Evidenzqualität mit ein (s. unten „Bewertung der Qualität der Evidenz“). Wenn in der Literatur keine Daten zu diesen Endpunkten identifiziert werden konnten, wurden die in den Studien berichteten Endpunkte analysiert und bewertet.

\section{Einschlusskriterien, Literatursuche und Datenextraktion}

Vor Beginn der Literatursuche wurden die Ein- und Ausschlusskriterien für die jeweilige Personengruppe entsprechend den PICO-Kriterien („Population, Intervention, Comparison, Outcome“) definiert. Zwei Autoren führten die Literatursuche unabhängig voneinander in verschiedenen Datenbanken durch und beurteilten die Relevanz von Titeln, Zusammenfassungen und Volltexten. Bei der Suche nach systematischen Reviews wurde in den Datenbanken MEDLINE, EMBASE und Cochrane Database of Systematic Reviews gesucht, bei der Durchführung eigener systematischer Reviews erfolgt die Suche in MEDLINE, EMBASE, im Cochrane Central Register of Controlled Trials und ggf. in weiteren Datenbanken (z. B. clinicaltrials.gov). Der Einschluss von Literatur erfolgte unabhängig von Sprache und Publikationsstatus, sog. graue Literatur (z.B. Kongressabstracts, Dissertationen) wurden ebenfalls berücksichtigt.

Sowohl bei identifizierten Reviews als auch bei eigenen systematischen Reviews erfolgte die Sichtung, Extraktion und Bewertung relevanter Daten aus den Originalstudien. Die Datenextraktion erfolgte durch zwei der Autoren unabhängig voneinander auf entsprechende Datenextraktionsformulare.

Bewertung des möglichen Verzerrungspotenzials eingeschlossener Studien

Zwei Autoren beurteilten unabhängig voneinander das mögliche Verzerrungspotenzial („risk of bias“) der individuellen Studien. Für RCTs wurde das „Risk of bias"-Instrument der Cochrane-Collaboration [28] und für Beobachtungsstudien die Newcastle-Ottawa-Skala bzw. das CASP („Critical Appraisal Skills Programme“)-Instrument [29] verwendet. Die Beurteilung des Verzerrungspotenzials jeder einzelnen Studie bzw. jedes 
einzelnen Endpunkts erfolgte als „hoch“, "niedrig“ oder ,unklar“.

\section{Bewertung der Qualität der Evidenz}

Entsprechend der SOP der STIKO erfolgte die Bewertung der Qualität der Evidenz mit der GRADE-Methodik [30, 31]. Bei der Bewertung der patientenrelevanten Endpunkte in den verschiedenen Indikationsgruppen wurden alle von der STIKO als „kritisch“ oder „wichtig" definierten Endpunkte für die Entscheidungsfindung beurteilt. Entsprechend den Vorschlägen der GRADE-Working Group wurden in erster Linie RCTs bewertet. Nur wenn diese nicht zur Verfügung standen oder mit einem hohen Verzerrungspotenzial behaftet waren, erfolgte eine Bewertung von Beobachtungsstudien.

\section{Statistische Analyse}

Soweit unter methodischen und inhaltlichen Aspekten möglich, wurden die Daten der jeweiligen Personengruppe in einer Metaanalyse zusammengefasst. War dies nicht möglich, erfolgte die Ergebnisdarstellung deskriptiv. Die statistischen Analysen erfolgten mit dem Review Manager (RevMan 5.2, Cochrane Collaboration, Kopenhagen, Dänemark), die Darstellung der GRADE-Evidenztabellen mit der GRADEpro Software [32].

\section{Ergebnisse}

Risiko für schwere Krankheitsverläufe in den Indikationsgruppen und bei Senioren bei Influenzainfektionen

Es wurden zwei systematischen Reviews $[10,33]$ sowie Daten einer Studie aus Deutschland [34] zur Beurteilung der Krankheitslast herangezogen.

Der systematische Review von Mertz et al. [10] untersuchte das Risiko für schwere Krankheitsverläufe in einer Vielzahl von Personengruppen, die an saisonaler oder pandemischer (2009) Influenza erkrankt waren. In dem Review wurden insgesamt 234 Beobachtungsstudien (97\% Kohortenstudien) mit über 600.000 Personen eingeschlossen. Die Mehrzahl der Studien wurde während der Pandemie 2009/10 durchge- führt ( $n=175)$. Laborbestätigte Endpunkte wurden in 95\% der während der Pandemie und in $84 \%$ der außerhalb der Pandemie durchgeführten Studien erhoben. Als relevante Endpunkte beschrieben wurden: ambulant erworbene Pneumonie, Mortalität jeglicher Ursache, Hospitalisierung jeglicher Ursache, Einlieferung auf eine Intensivstation (ICU) sowie Beatmungspflicht.

Bezüglich saisonaler Influenzaerkrankungen zeigte sich bei Vorliegen eines Risikofaktors bzw. einer Komorbidität (,any risk factor/any comorbidity") eine erhöhtes Risiko für Pneumonie (Odds ratio [OR]: 1,5, $95 \%$ Konfidenzintervall [95\% KI]: 1,0-2,2), Hospitalisierung (OR: 3,4, $95 \%$ KI: 2,6-4,4), Einlieferung auf eine Intensivstation (ICU; OR: 1,7, $95 \%$ KI: 1,3-2,3) oder Mortalität (OR: 2,0, $95 \%$ KI: 1,7-2,4).

Eine nach Risikogruppen stratifizierte Darstellung der Daten aus dem Review von Mertz et al. [10] war aufgrund der Studienlage nur eingeschränkt möglich:

- Bei HIV-positiven Personen untersuchte nur eine der in dem Review eingeschlossenen Studien eine mögliche Assoziation zwischen einer saisonalen Influenzainfektion und Mortalität jeglicher Ursache. Ein statistisch signifikanter Effekt konnte, bei kleinen Fallzahlen, nicht gezeigt werden (OR: 3,9, 95 \% KI: 0,52-29,0).

- In 2 Studien konnte Diabetes mellitus als Risikofaktor für eine Hospitalisierung (OR: 9,1, $95 \% \mathrm{KI}$ : 5,5-18,0), nicht jedoch für Pneumonien (OR 0,91, $95 \%$ KI: 0,26-3,24) oder für Mortalität jeglicher Ursache $(0,59$, $95 \%$ KI: 0,23-1,5) identifiziert werden.

- Bei Personen mit Asthma zeigte 1 Studie eine statistisch signifikante Assoziation zwischen einer Influenzainfektion und Pneumonie (OR: 1,5, $95 \%$ KI: 1,1-1,6). Für ICU-Einweisungen (OR: 1,4, $95 \%$ KI: 0,3-6,8) oder Mortalität jeglicher Ursache (OR: 0,89, 95\% KI: 0,1-7,7) fanden sich keine erhöhten Risiken.

- Bei Personen mit kardiovaskulären Erkrankungen war die Datenlage mit bis zu 8 Studien pro Endpunkt umfangreicher. Demnach stellten bei einer Influenzainfektion kardiovaskuläre Erkrankungen einen Risikofaktor für Pneumonien (OR: 1,6, 95 \% KI: 1,1-2,3), Beatmungspflicht (OR: 3,3,
95\% KI: 1-10,6) und Mortalität (OR: $2,0,95 \% \mathrm{KI}: 1,1-3,7)$, nicht jedoch für ICU-Einweisungen dar.

- Bei Schwangeren konnte in 2 Studien kein statistisch signifikanter Zusammenhang zwischen einer saisonalen Influenzainfektion und Mortalität jeglicher Ursache identifiziert werden (OR: 1,1, 95\% KI: 0,8-1,5). Daten zu anderen Endpunkten wurden nicht berichtet.

- Senioren mit einer Influenzainfektion hatten im Vergleich zu jüngeren Erwachsene ein erhöhtes Risiko für eine Hospitalisierung (OR: 4,7, $95 \% \mathrm{KI}$ : 1,7-12,4) und ein erhöhtes Risiko zu versterben (OR: 3, $95 \%$ KI: 1,5-5,7).

Bezüglich pandemischer Influenza wurden in dem Review von Mertz et al. [10] deutlich mehr Studien identifiziert und eingeschlossen. Hier fand sich bei Vorliegen eines Risikofaktors bzw. einer Komorbidität (,any risk factor/any comorbidity") in bis zu 53 Studien pro Endpunkt eine Assoziation mit Hospitalisierung (OR: 2,7, $95 \%$ KI: 1,9-4,0), Einlieferung auf eine ICU (OR: 1,9, 95\% KI: 1,6-2,4) und Tod jeglicher Ursache (OR: 2,0, $95 \%$ KI: 1,7-2,4). Kein Zusammenhang konnte für die Endpunkte Pneumonie oder Beatmungspflicht beobachtet werden.

Eine nach Risikogruppen stratifizierte Analyse zeigte folgende Ergebnisse:

- Bei HIV-positiven Personen wurden keine statistisch signifikanten Effekte bezüglich der erhobenen Endpunkte identifiziert. Eine (nicht näher definierte) "Immunsuppression“ war hingegen ein Risikofaktor für Hospitalisierung (OR: 4,6, 95\% KI: 2,4-8,8) und Mortalität (OR: 3,7, 95\% KI: 1,87,6), nicht jedoch für die Einlieferung auf eine ICU oder für eine Pneumonie.

- Personen mit Diabetes mellitus und einer pandemischen Influenzainfektion wurden im Vergleich zu Personen ohne Grunderkrankung mit einer höheren Wahrscheinlichkeit hospitalisiert (OR: 4,3, $95 \%$ KI: 3,1-5,8). Ebenso erhöht waren die ORs für eine ICU-Einlieferung (OR: 1,6, $95 \% \mathrm{KI}$ : 1,3-1,9) und Mortalität (OR: 2,2, $95 \%$ KI: 1,4-3,6), nicht jedoch für Pneumonien (OR: 0,56, $95 \%$ KI: 0,1-2,6).

- Bei Personen mit Asthma fand sich kein erhöhtes Risiko für die in dem 


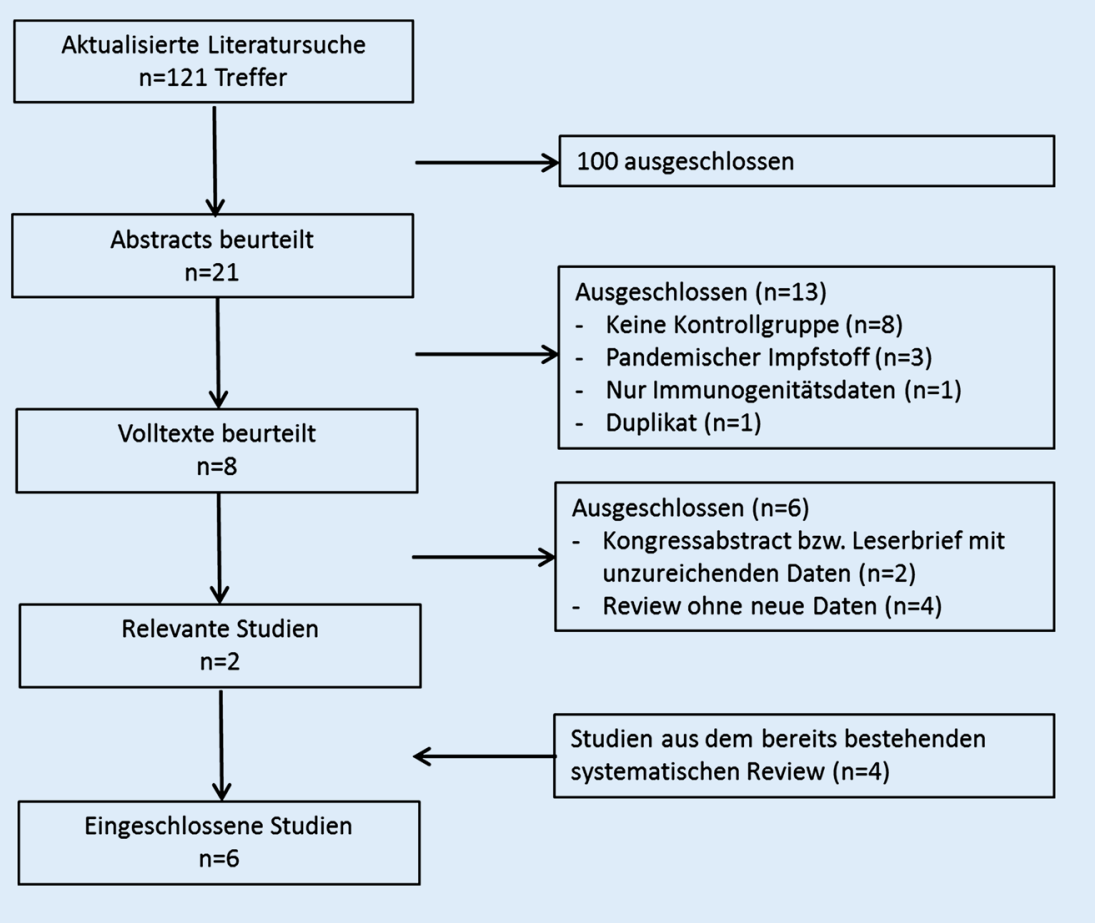

Abb. 1 ॥ Flussdiagramm für die aktualisierte systematische Literatursuche zur Effektivität und Sicherheit der Influenzaimpfung bei HIV-positiven Personen

Review evaluierten Endpunkte. Die Punktschätzer (OR) lagen dabei je nach Endpunkt zwischen 0,83 (95\% KI: 0,6-1,2) für ICU-Einlieferung und 1,9 (95\% KI: 0,9-4,1) für den Endpunkt Pneumonie.

- Personen mit kardiovaskulären Erkrankungen hatten bei Infektion mit dem pandemischen Influenzavirus eine erhöhte Wahrscheinlichkeit für eine Hospitalisierung (OR: 3,5, $95 \%$ KI: 2,3-5,5), ICU-Einlieferung (OR: $1,7,95 \% \mathrm{KI}: 1,4-2,1)$ und zu versterben (OR: 2,9, $95 \%$ KI: 1,8-4,9).

- Eine Schwangerschaft war während der Pandemie 2009 ein Risikofaktor für eine Hospitalisierung (OR: 3,5, $95 \% \mathrm{KI}: 1,7-7,4)$, hingegen war das Risiko für eine ICU-Einlieferung nach den vorliegenden Daten reduziert (OR: 0,62, 95\% KI: 0,52-0,75).

- Höheres Alter war wie bei der saisonalen Influenza ein Risikofaktor für eine Hospitalisierung (OR: 2,8, $95 \%$ KI: 1,8-4,6) und für Mortalität (OR: 2,7, $95 \%$ KI: 1,5-4,7).

In einer Studie der WHO wurde anhand individueller Studiendaten aus 19 Ländern das Risiko für eine schwere Erkran- kung und Tod bei Personen mit A(H1N1) pdm09-Infektionen untersucht [33]. Dazu wurden Daten von mehr als 80.000 laborbestätigten Influenzaerkrankten herangezogen. Die Autoren fanden, dass das Vorliegen einer Immunsuppression, von Asthma, einer kardialen Vorerkrankung, einer Nieren- oder Lebererkrankung oder einer neurologischen Erkrankung das Risiko einer Hospitalisierung um den Faktor 2 bis 24 und das Risiko zu Versterben um den Faktor 2 bis 28 erhöhte. Personen mit Diabetes oder neurologischen Erkrankungen zeigten in dieser Studie kein erhöhtes Risiko für eine Hospitalisierung, jedoch ein 4- bis 17-fach erhöhtes Risiko zu versterben. Schwangere hatten ein knapp 7-fach erhöhtes Risiko für eine Hospitalisierung (RR: 6,8, 95\% KI: 4,5-12,3).

Eine Studie aus Deutschland analysierte die gemäß Infektionsschutzgesetz gemeldeten laborbestätigten $\mathrm{A}(\mathrm{H} 1 \mathrm{~N} 1)$ pdm09-Fälle während der Influenzapandemie 2009 [34]. In diese Studie wurden insgesamt über 170.000 Einzelfälle, davon knapp 7700 Hospitalisierungen und 250 Todesfälle, eingeschlossen. In allen Altersgruppen hatten Personen mit einer Immunsuppression, Diabetes mellitus, einer Herz-Kreislaufoder einer Atemwegserkrankung eine hö- here Wahrscheinlichkeit für eine Hospitalisierung, verglichen mit Personen ohne Grunderkrankung. Die Punktschätzer (OR) lagen je nach Vorerkrankung in der Altersgruppe der 0- bis 14-Jährigen zwischen 1,9 und 7,5, bei den 15- bis 59-Jährigen zwischen 2,3 und 11,0 und bei Senioren zwischen 2,3 und 9,4. Auch bei Schwangeren war im Vergleich zu Nichtschwangeren die Wahrscheinlichkeit für eine Hospitalisierung deutlich erhöht (OR: 9,3, 95\% KI: 7,5-11,4). Bis auf die Gruppe der Schwangeren war das Risiko zu versterben in allen oben genannten Risikogruppen erhöht.

\section{Zusammenfassung und Beurteilung}

Bei Vorliegen einer Grunderkrankung im Sinne der STIKO-Indikationsgruppen, bei Schwangerschaft und in höherem Alter besteht ein erhöhtes Risiko für schwere Krankheitsverläufe, z. B. gemessen als Hospitalisierung, Entwicklung einer Pneumonie oder Einlieferung auf eine Intensivstation.

\section{Effektivität und Sicherheit der Influ- enzaimpfung}

Zur Identifikation bereits bestehender systematischen Reviews zur Wirksamkeit und Sicherheit der Influenzaimpfung in Indikationsgruppen bzw. bei Senioren wurde eine systematische Suche im Zeitraum von 1990-2013 durchgeführt [35]. Von initial 564 identifizierten Studien wurden 46 potenziell relevante systematische Reviews identifiziert und die methodische Qualität der Reviews mittels AMSTAR [26] bewertet. Hieraus ergab sich, dass systematische Reviews zu folgenden Personengruppen verwendet werden konnten: HIV-positiven Personen, Personen mit chronischen Lungenerkrankungen, Personen mit kardiovaskulären Erkrankungen, medizinisches Personal, Schwangere und Senioren. Für Personen mit Diabetes mellitus bzw. Personen mit (terminaler) Niereninsuffizienz waren keine Reviews verfügbar.

\section{HIV-positive Personen}

Als Grundlage für diese Indikationsgruppe wurde der 2006 publizierte systematische Review von Atashili et al. [36] (AMSTAR-Score 10/11) verwendet und mittels einer systematischen Literatursu- 
Tab. 1 Basischarakteristika der in den systematischen Review eingeschlossenen Originalstudien zur Wirksamkeit und Sicherheit der Influenzaimpfung bei HIV-positiven Personen

\begin{tabular}{|c|c|c|c|c|c|c|}
\hline Autor, Jahr & Land & $\begin{array}{l}\text { Studien } \\
\text { design }\end{array}$ & $\begin{array}{l}\text { Alter (MW oder } \\
\text { Spannweite) }\end{array}$ & $\begin{array}{l}\text { Anzahl Studien- } \\
\text { teilnehmer }\end{array}$ & $\begin{array}{l}\text { CD4-Zellzahl/ } \mu \mathrm{l} \\
\text { bei Geimpften }\end{array}$ & $\begin{array}{l}\text { CD4-Zellzahl/ } \mu \mathrm{l} \\
\text { bei Ungeimpften }\end{array}$ \\
\hline \multicolumn{7}{|l|}{ Erwachsene } \\
\hline Tasker et al. 1999 [39] & USA & $\mathrm{RCT}$ & 33 Jahre & 102 & $\begin{array}{l}\text { MW: } 398 \text { (SD: 170), } \\
10 \%<200\end{array}$ & $\begin{array}{l}\text { MW: } 409 \text { (SD: 185), } \\
17 \%<200\end{array}$ \\
\hline Fine et al. 2001 [42] & USA & Kohorte & 38-42 Jahre & 71 & $8 \% \leq 100$ & $28 \% \leq 100$ \\
\hline Ranieri et al. 2005 [40] & Italien & Kohorte & 20-69 Jahre & 145 & $13 \%<200^{a}$ & $29 \%<200^{a}$ \\
\hline Yamanaka et al. 2005 [41] & Japan & Kohorte & 40-41 Jahre & 332 & $19 \%<200$ & $14 \%<200$ \\
\hline Madhi et al. 2011 [38] & Südafrika & $\mathrm{RCT}$ & 34 Jahre & 406 & Median $273^{a}$ & Median $290^{\mathrm{a}}$ \\
\hline \multicolumn{7}{|l|}{ Kinder } \\
\hline Madhi et al. 2013 [43] & Südafrika & $\mathrm{RCT}$ & 23,8 Monate & 410 & Median $1770^{\mathrm{a}}$ & $1648^{\mathrm{a}}$ \\
\hline
\end{tabular}

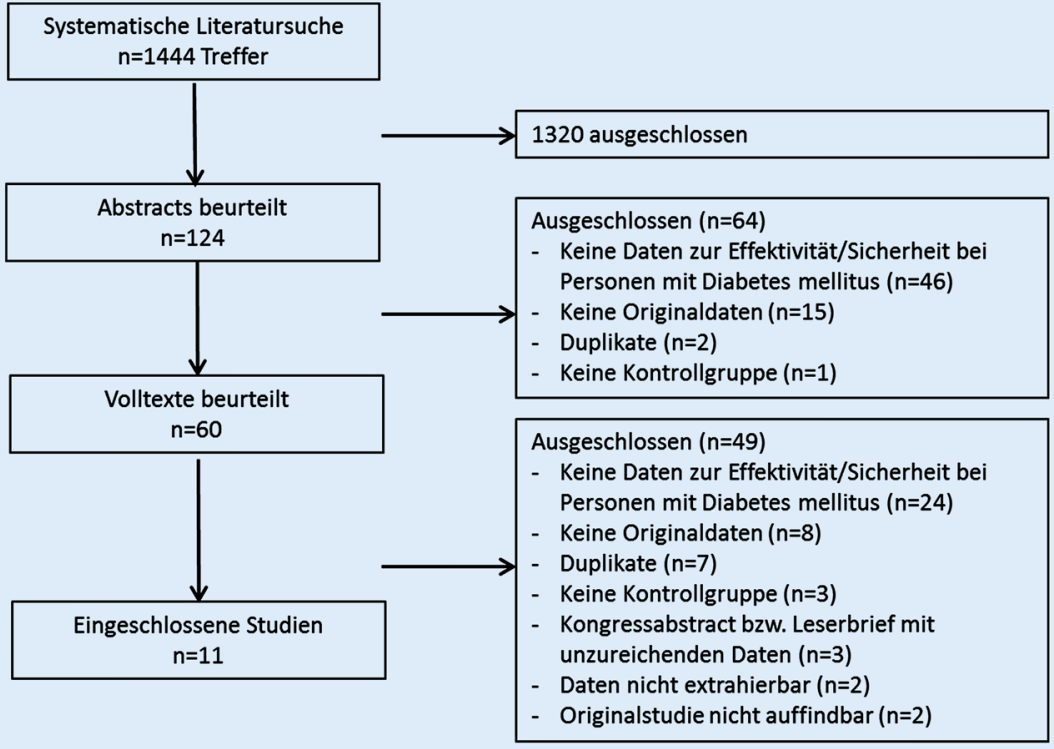

Abb. 2 \ Flussdiagramm für die systematische Literatursuche zur Effektivität und Sicherheit der Influenzaimpfung bei Personen mit Diabetes mellitus

che aktualisiert [37]. Hierdurch konnte die Evidenzbasis des Reviews von Atashili et al. [36] um 2 auf insgesamt 6 Originalstudien (3 RCTs und 3 Beobachtungsstudien) erweitert werden (• Abb. 1).

Bezüglich Erwachsenen wurden 2 RCTs (Südafrika, USA $[38,39]$ ) und 3 Beobachtungsstudien (Italien, Japan, USA [40-42]) eingeschlossen, die zwischen 1995 und 2008 durchgeführt wurden. Bezüglich Kindern konnte 1 RCT eingeschlossen werden, der bei HIVpositiven Kindern im Alter von 6-59 Monaten (2009, Südafrika [43]) durchgeführt wurde (• Tab. 1). Insgesamt wurden 1562 HIV-positiven Personen eingeschlossen und Ergebnisse zu 6 Endpunkten beschrieben. In allen Studien wurden trivalente, inaktivierte Impfstoffe (TIV) verwendet. Das Verzerrungspotenzial wurde in den RCTs als niedrig und in den Beobachtungsstudien als

Bei HIV-positiven Erwachsenen fand sich eine Impfeffektivität (VE) gegen laborbestätigte Influenzainfektionen von 85\% (95\% KI: 22-97\%). Bei 5-59 Monate alten Kindern zeigte sich kein signifikanter Effekt, die VE gegen laborbestätigte Influenzainfektionen lag bei $11 \%(95 \% \mathrm{KI}$ : hoch beurteilt.
$-70-54 \%)$. Weder bei Kindern noch bei Erwachsenen zeigten sich statistisch signifikante Effekte bei anderen Endpunkten (Mortalität, Hospitalisierung oder Pneumonie jeglicher Ursache und ILI). Dies was vorrangig durch kleine Fallzahlen bedingt und führte bei diesen Endpunkten auch zu einer Herabstufung der Evidenzqualität um 2 Punkte (von „hoch" auf „niedrig") (die GRADE-• Tab. 1a und 1b sind online als elektronisches Zusatzmaterial beim Bundesgesundheitsblatt unter der Bezeichnung „Anhang zum Hintergrundpapier der STIKO“ abrufbar).

Daten zur Sicherheit lieferten die 2 RCTs aus Südafrika: Hier fanden sich keine Hinweise auf schwerwiegende unerwünschte Arzneimittelwirkungen (UAW).

\section{Zusammenfassung und Beurteilung}

Die Studien bei HIV-positiven Erwachsenen zeigen, dass die TIV-Influenzaimpfung effektiv Influenzainfektionen verhindert (Evidenzqualität: moderat). Bei Kindern im Alter von 5-59 Monaten wurde gegen keinen der berichteten Endpunkte eine statistisch signifikante Effektivität gemessen (Evidenzqualität: moderat).

\section{Personen mit Diabetes mellitus}

Da für Personen mit Diabetes mellitus kein systematischer Review vorlag, wurde ein eigener systematischer Review durchgeführt [44]. Die Literatursuche ergab 1444 Treffer, von denen 11 Studien in die Analyse eingeschlossen werden konnten (• Abb. 2). 
Tab. 2 Basischarakteristika der in den systematischen Review eingeschlossenen Originalstudien zur Wirksamkeit und Sicherheit der Influenzaimpfung bei Personen mit Diabetes

\begin{tabular}{|c|c|c|c|c|}
\hline Autor, Jahr & Land & Studiendesign & $\begin{array}{l}\text { Alter } \\
\text { (MW oder Spannweite) }\end{array}$ & $\begin{array}{l}\text { Anzahl } \\
\text { Studienteilnehmer }\end{array}$ \\
\hline Colquhoun et al. 1997 [52] & UK & Fall-Kontroll & bis 85 Jahre & 114 \\
\hline Gasparini et al. 2013 [53] & Italien & Fall-Kontroll & nicht berichtet & 78 \\
\hline Hak et al. 2002 [45] & USA & Kohorte & $\geq 65$ Jahre & 21.991 \\
\hline Heymann et al. 2004 [46] & Israel & Kohorte & 73 Jahre & 16.383 \\
\hline Isotani 2000 [47] & Japan & Kohorte & Nicht berichtet & 450 \\
\hline Lau et al. 2013 [54] & Kanada & Fall-Kontroll & $\begin{array}{l}\text { Median für } 2 \text { Subgruppen: } \\
53 \text { bzw. } 74 \text { Jahre }\end{array}$ & 91.605 \\
\hline Looijmans et al. 2006 [55] & Niederlande & Fall-Kontroll & 69 Jahre & 1753 \\
\hline Rodriguez-Blanco et al. 2012 [48] & Spanien & Kohorte & 74 Jahre & 2650 \\
\hline Selvais et al. 1997 [50] & Belgien & Kohorte & 56 Jahre & 432 \\
\hline Schade und McCombs 2000 [49] & USA & Kohorte & 65-114 Jahre & 26.443 \\
\hline Wang et al. 2013 [56] & Taiwan & Fall-Kontroll & 73 Jahre & 9025 \\
\hline
\end{tabular}

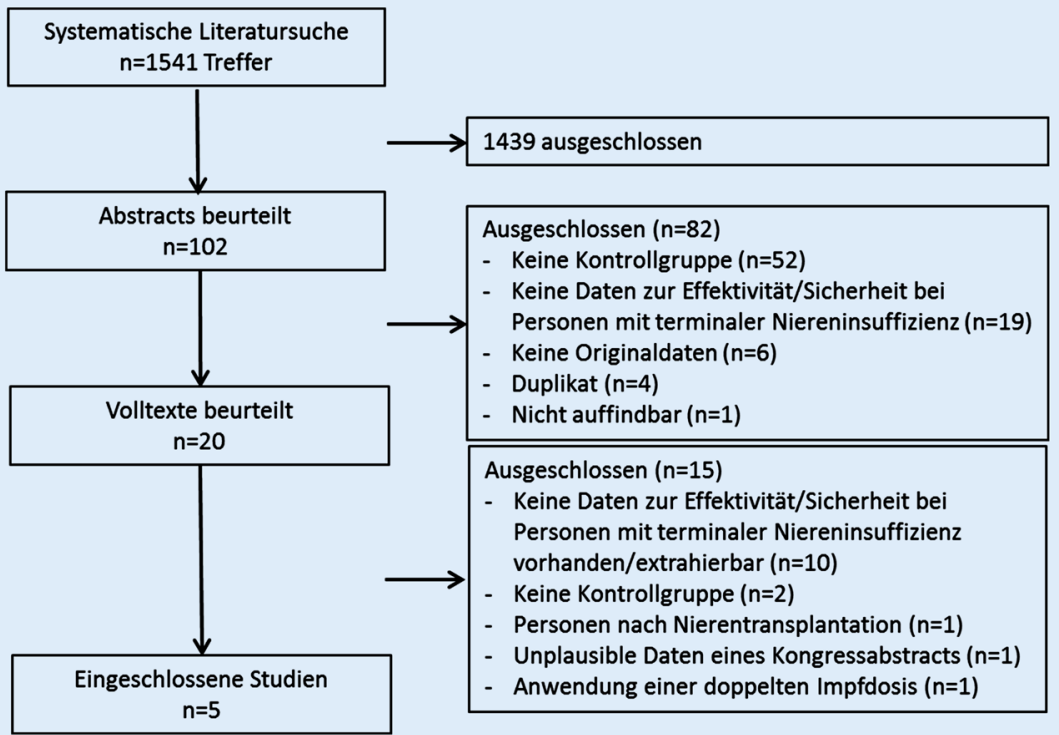

Abb. 3 ॥ Flussdiagramm für die systematische Literatursuche zur Effektivität und Sicherheit der Influenzaimpfung bei Personen mit terminaler Niereninsuffizienz

Alle eingeschlossenen Studien waren Beobachtungsstudien (6 Kohorten- [4550] und 5 Fall-Kontroll-Studien [51-56]), die in Europa $(n=5)$, Nordamerika $(n=3)$ und in Asien $(n=3)$ durchgeführt wurden und insgesamt 170.924 Studienteilnehmer mit Diabetes mellitus einschlossen (- Tab. 2). In 9 Studien wurden klinische Diagnosecodes (z.B. ICD-9/10-Codes) zur Identifikation der Endpunkte verwendet. Laborbestätigte Endpunkte wurden nicht beschrieben. In 6 Studien wurden und eine VE von $43 \%$ (95\% KI: 28-54\%) gegen P/I-Hospitalisierungen. Die VE gegen Mortalität und influenzaähnliche Erkrankungen waren in dieser Altersgruppe statistisch nicht signifikant. Bei Senioren (65+) zeigte die Impfung signifikante Effekte gegen alle berichteten Endpunkte mit einer VE zwischen 13\% (95\% KI: 10-16\%) gegen ILI und 56\% (95\% KI: 47-64\%) gegen Mortalität jeglicher Ursachen.

$\mathrm{Da}$ in keiner der Studien laborbestätigte Endpunkte analysiert wurden, müssen die Ergebnisse zurückhaltend interpretiert werden. Je nach Zusammensetzung der Studienpopulation kann es zu einer Über-, aber auch zu einer Unterschätzung der Impfeffektivität kommen. So wurden beispielsweise in einer Beobachtungsstudie statistisch signifikante Effekte der Impfung außerhalb der Influenzasaison gemessen. Da außerhalb der Influenzasaison ein schützender Effekt aufgrund einer niedrigen oder fehlenden Viruszirkulation nicht plausibel ist, scheint es sich hier um eine „Pseudoeffektivität“ $\mathrm{zu}$ handeln. Eine mögliche Erklärung für diese Verzerrung ist, dass die geimpften Teilnehmer in dieser Studie gesünder waren als die Ungeimpften und damit per se ein niedrigeres Risiko für einen der unspezifischen Endpunkte hatten ("healthy vaccinee bias"). Dieser "healthy vaccinee bias“ kann dann zu einer Überschätzung der VE führen.

Trotz des Verzerrungspotenzials kann aus den Daten nicht gefolgert werden, dass die Influenzaimpfung bei Personen mit Di- 
Tab. 3 Basischarakteristika der in den systematischen Review eingeschlossenen Originalstudien zur Wirksamkeit und Sicherheit der Influenzaimpfung bei Personen mit terminaler Niereninsuffizienz

\begin{tabular}{|c|c|c|c|c|}
\hline Autor, Jahr & Land & Studiendesign & $\begin{array}{l}\text { Alter } \\
\text { (MW oder Spannweite) }\end{array}$ & $\begin{array}{l}\text { Anzahl } \\
\text { Studienteilnehmer }\end{array}$ \\
\hline Bond et al. 2012 [58] & USA & Kohorte & 59 & 21.991 \\
\hline Gilbertson et al.2003 [59] & USA & Kohorte & $40-64$ & 16.383 \\
\hline McGrath et al. 2012 [60] & USA & Kohorte & 63 & 450 \\
\hline Slinin et al. 2006 [61] & USA & Kohorte & 60 & 432 \\
\hline Wang et al. 2013 [62] & Taiwan & Kohorte & 59-70 Jahre & 9025 \\
\hline
\end{tabular}

abetes unwirksam ist. Denn 1) konnte ein Verzerrungspotenzial nicht in allen Studien gleichermaßen nachgewiesen werden, 2) zeigten die Studien bei der Mehrzahl der Endpunkte protektive Effekte der Impfung und 3) erscheint eine fehlende Wirksamkeit der Influenzaimpfung bei Personen mit Diabetes mellitus biologisch nicht plausibel.

Die Qualität der Evidenz lag bei allen klinischen Endpunkten bei sehr niedrig bis niedrig und war vor allem durch das Fehlen von RCTs und das hohe Verzerrungsrisikos bei einigen Endpunkten bedingt (die GRADE- $\bullet$ Tab. 2a und $2 \mathbf{b}$ sind online als elektronisches Zusatzmaterial beim Bundesgesundheitsblatt unter der Bezeichnung „Anhang zum Hintergrundpapier der STIKO“ abrufbar).

Daten zur Sicherheit der Impfung wurden in den Studien nicht beschrieben. Eine zusätzlich durchgeführte orientierende Literatursuche nach Fallserien und Fallberichten erbrachte keine Hinweise auf schwerwiegende UAWs in dieser Personengruppe.

\section{Zusammenfassung und Beurteilung}

Die existierenden Daten weisen darauf hin, dass die saisonale Influenzaimpfung bei Erwachsenen mit Diabetes mellitus gegen unspezifische Endpunkte (Hospitalisierung, Tod) schützen kann. Allerdings sind die Effektschätzer mit großer Unsicherheit behaftet (Evidenzqualität sehr niedrig bis niedrig), insbesondere weil Studien mit laborbestätigten Endpunkten fehlen.

\section{Personen mit terminaler Niereninsuffizienz}

Mittels Literatursuche wurden 1541 potenziell relevante Studien identifiziert, von denen 5 in die Analyse eingingen (• Abb. 3, [57]).

Bei allen eingeschlossenen Originalstudien handelte es sich um Kohortenstudien, die in den USA ( $n=4$ [58-61]) und in Taiwan ( $n=1$ [62]) bei Erwachsenen mit terminaler Niereninsuffizienz durchgeführt wurden (- Tab. 3). Von den insgesamt 9 berichteten Endpunkten wurde Mortalität jeglicher Ursache, Hospitalisierung jeglicher Ursache und Hospitalisierungen aufgrund einer Pneumonie oder Influenza (P/I-Hospitalisierung) in mehr als einer Studie beschrieben. Alle Endpunkte wurden mittels Krankheitsdiagnosecodes (z. B. ICD-10-Codes) identifiziert und waren influenzaunspezifisch (d.h. nicht laborbestätigt).

Die VE betrug 32\% (95\% KI: 24$39 \%)$ gegen Mortalität und 14\% (95\% KI: 7-20\%) gegen P/I-Hospitalisierungen. Der Effekt der Impfung gegen Hospitalisierungen jeglicher Ursachen war statistisch nicht signifikant (VE: 12\%, $95 \% \mathrm{KI}$ : -4-26\%). Ähnlich wie bei dem systematischen Review zu Personen mit Diabetes mellitus wurden auch hier in einzelnen Studien Pseudoeffektivitäten außerhalb der Influenzasaison gegen verschiedene Endpunkte gemessen.

Die Qualität der Evidenz wurde aufgrund des hohen Verzerrungspotenzials für alle Endpunkte als „sehr niedrig“ bewertet (GRADE- Tab. 3 ist online als elektronisches Zusatzmaterial beim Bundesgesundheitsblatt unter der Bezeichnung „Anhang zum Hintergrundpapier der STIKO“ abrufbar).

Daten zur Sicherheit der Impfung wurde in den identifizierten Studien nicht beschrieben, jedoch fanden sich in einer zusätzlichen, orientierenden Literatursu- che keine Hinweise auf schwerwiegende UAWs.

\section{Zusammenfassung und Beurteilung}

Die existierenden Studien zeigen bei Personen mit terminaler Niereninsuffizienz eine Wirksamkeit der saisonalen Influenzaimpfung gegen unspezifische Endpunkte (Tod, Hospitalisierung, ILI). Die insgesamt sehr niedrige Evidenzqualität ist auf das Fehlen von RCTs bzw. Studien, die laborbestätigte Endpunkte verwenden, zurückzuführen.

\section{Personen mit chronischen Lungenerkrankungen}

Für diese Indikationsgruppe wurde der Cochrane-Review von Cates et al. (AMSTAR-Score 8/11) verwendet, der die Wirksamkeit und Sicherheit der Influenzaimpfung bei Personen mit Asthma untersucht hat [63]. Aus diesem Review, der insgesamt 18 RCTs einschloss, wurden nur solche Originalstudien berücksichtigt, die TIV mit einer Placebogruppe verglichen. RCTs, in denen mono- oder bivalente Impfstoffe verwendet $(n=2)$, verschiedene Influenzaimpfstoffe miteinander verglichen $(n=3)$, ein atypisches Impfschema angewendet $(n=1)$ oder in denen Lebendimpfstoffe eingesetzt wurden $(n=3)$, gingen in die vorliegende Analyse nicht ein. Von den verbleibenden 9 RCTs machten 4 Studien Angaben zu den folgenden von der STIKO a priori festgelegten patientenrelevanten Endpunkten [51, 64-67]: Influenzabedingte Asthmaexazerbationen (laborbestätigt), Asthmaexazerbationen (jeglicher Schwergrad), Hospitalisierung wegen Asthmas, Pneumonie sowie asthmabedingte Arztkonsultationen. 


\begin{tabular}{|c|c|c|c|c|c|c|c|c|c|}
\hline Studie & \multicolumn{2}{|c|}{ Studiengruppe } & \multicolumn{2}{|c|}{ Kontrolle } & \multicolumn{2}{|c|}{$\begin{array}{c}\text { Relatives Risiko } \\
\text { tt Gewichtung M-H, random, } 95 \% \text {-KI }\end{array}$} & Jahr & \multicolumn{2}{|c|}{$\begin{array}{c}\text { Relatives Risiko } \\
\mathrm{M}-\mathrm{H} \text {, random, } 95 \%-\mathrm{KI}\end{array}$} \\
\hline FLUVACS 2004 & 32 & 145 & 54 & 147 & $49,6 \%$ & $0,60[0,41,0,87]$ & 2004 & 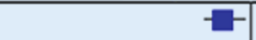 & \\
\hline FLUCAD 2008 & 16 & 325 & 30 & 333 & $19,9 \%$ & $0,55[0,30,0,98]$ & 2008 & -1 & \\
\hline IVCAD 2009 & 3 & 135 & 3 & 131 & $2,7 \%$ & $0,97[0,20,4,72]$ & 2009 & & \\
\hline Phrommintikul 2011 & 20 & 221 & 42 & 218 & $27,7 \%$ & $0,47[0,29,0,77]$ & 2011 & $\rightarrow$ & \\
\hline Gesamt (95\%-KI) & & 826 & & 829 & $100,0 \%$ & $0,56[0,43,0,73]$ & & 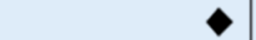 & \\
\hline Ereignisse gesamt & 71 & & 129 & & & & & & \\
\hline $\begin{array}{l}\text { Heterogenität: Tau }{ }^{2}= \\
\text { Test auf Gesamteffek }\end{array}$ & $\begin{array}{l}00 ; \mathrm{Chi}^{2}= \\
Z=4,36(P\end{array}$ & $\begin{array}{l}09, \mathrm{df}= \\
0,0001\end{array}$ & $3(P=0$, & 78); $1^{2}=$ & $=0 \%$ & & gunsten & $\begin{array}{cc}0,01 & 0,1 \\
\text { Interventionsgruppe }\end{array}$ & $\begin{array}{cc}10 & 100 \\
\text { Zugunsten Kontrolle }\end{array}$ \\
\hline
\end{tabular}

Abb. $4 \Delta$ Effektivität der Influenzaimpfung gegen schwerwiegende kardiovaskuläre Ereignisse (MACE (major adverse cardiac events)) bei Personen mit kardiovaskulären Vorerkrankungen. KI Konfidenzintervall, $M$ - $H$ Mantel-Haenszel

In den 5 RCTs aus Europa $(n=4)$ und den USA $(n=1)$ wurden insgesamt 3246 Studienteilnehmern mit Asthma eingeschlossen. Ein RCT schloss Kinder im Alter von 8-18 Jahren ein. Das Verzerrungsrisiko war bei allen 5 RCTs niedrig. Daten zur Impfeffektivität berichtete nur Bueving et al. [64], Daten zur Sicherheit wurde in 3 RCTs erhoben. Aus der verbleibenden Studie von Goevert et al. [65] konnten die Daten von Personen mit Asthma nicht extrahiert werden.

In dem RCT zur Impfeffektivität [64], in dem 8-18-jährige Kinder über 2 Saisons (H3N2 und H1N1 als dominante Viren) beobachtet wurden, konnten keine protektiven Effekte der Impfung nachgewiesen werden: Die VE gegen influenzabedingte Asthmaexazerbationen lag bei $-31 \%$ (95\% KI: $-161-34 \%$ ) und gegen Asthmaexazerbationen jeglichen Schweregrades bei $0 \%$ (95\% KI: $-12-12 \%)$. Allerdings war die Dauer der Asthmaexazerbationen bei geimpften Kindern kürzer als bei ungeimpften Kindern. Hospitalisierungen aufgrund von Asthma oder Pneumonien traten in dem Studienzeitraum nicht auf. Die Autoren des RCTs diskutieren, ob die Ergebnisse auf eine unzureichende IgA-Antwort (Immunglobulin A) auf den in der Studie verwendeten TIV zurückzuführen ist und ob die Studiengröße ( $n=696$ Kinder) möglicherweise nicht ausreichend war.

In den übrigen 3 RCTs wurden die Studienteilnehmer nur über einen Zeitraum von 14 Tagen nach der Impfung beobachtet. Daher sind nur Angaben zur Sicherheit der Impfung, nicht jedoch zur Wirksamkeit möglich. Eine Studie [67] fand eine erhöhte Rate von Asthmaexazerbati- onen nach der Impfung (Relatives Risiko: $3,7,95 \%$ KI: 1,05-13,2), dies wurde in den anderen RCTs nicht bestätigt. Insgesamt fanden sich nach der Influenzaimpfung im Vergleich zu Placebo keine erhöhten Raten von asthmabedingten Arztkonsultationen, kein höherer Medikamentengebrauch und keine zusätzliche Einschränkung der Lungenfunktion.

\section{Zusammenfassung und Beurteilung}

Es gibt keine Hinweise auf schwerwiegende UAWs von Influenzatotimpfstoffen bei Personen mit Asthma bronchiale (Evidenzqualität niedrig bis moderat, GRADE- - Tab. 4a und $4 \mathrm{~b}$ sind online als elektronisches Zusatzmaterial beim Bundesgesundheitsblatt unter der Bezeichnung „Anhang zum Hintergrundpapier der STIKO“ abrufbar).

Die Datenlage zur Wirksamkeit von TIV bei Personen mit Asthma ist mit einem RCT (bei Kindern) äußerst begrenzt. Hier konnten keine statistisch signifikante Effekte gegen influenzabedingte Asthmaexazerbationen oder Asthmaexazerbationen jeglicher Art gezeigt werden (Evidenzqualität moderat bis hoch).

\section{Personen mit chronischen Herz- Kreislauf-Krankheiten}

Als Grundlage diente hier der Review von Udell et al. von 2013 (AMSTAR-Score 9/11) [68]. In diesem Review wurde die Effektivität bzw. die Sicherheit der Influenzaimpfung hinsichtlich kardiovaskulärer Ereignisse untersucht. Von 12 in dem Review eingeschlossenen RCTs wurden 6 RCTs, in denen die Effektivität bzw. Sicherheit von TIV im Vergleich mit einer
Placebo- oder einer Kontrollgruppe verglichen wurde, evaluiert. Allerdings mussten 2 der 6 RCTs $[65,69]$ ausgeschlossen werden, in denen nur $14 \%$ bzw. $16 \%$ der untersuchten Studienpopulation kardiovaskuläre Vorerkrankungen aufwiesen und somit nicht der STIKO-Indikationsgruppe entsprachen. Für die verbleibenden vier in Argentinien, Polen, Iran und Thailand durchgeführten RCTs wurde das Verzerrungsrisiko in einer Studie als hoch, in einer weiteren als niedrig und in zwei Studien als unklar bewertet.

Als kombinierter primärer Endpunkt wurden in dem systematischen Review schwerwiegende kardiovaskuläre Ereignisse („Major Adverse Cardiac Events“, MACE) zusammengefasst. Dieser beinhaltete folgende Endpunkte: Tod durch ein kardiovaskuläres Ereignis, Hospitalisierung aufgrund eines Herzinfarkts, instabile Angina pectoris, Schlaganfall, Herzinsuffizienz oder eine akut notwendige Koronarintervention. Als sekundäre Endpunkte wurden die genannten kardiovaskulären Ereignisse einzeln bewertet. Laborbestätigte Endpunkte wurden nicht berichtet.

Die Analyse der 4 RCTs umfasste 1655 Studienteilnehmer. Die VE gegen den primären Endpunkt (MACE) betrug 44\% (95\% KI: 27-57\%, - Abb. 4).

Die Impfeffektivität hinsichtlich der sekundären Endpunkte „kardiovaskuläre Mortalität“ (VE: 60\%, 95\% KI: 29-77\%) und „Mortalität jeglicher Ursache“ (VE: $54 \%$, $95 \%$ KI: 23-73) war ebenfalls statistisch signifikant, während bei den nichttödlichen sekundären Endpunkten keine protektiven Effekte nachgewiesen wurden. Eine stratifizierte Analyse zeigte, dass bei 
Tab. 4 Wirksamkeit und Sicherheit der Influenzaimpfung bei schwangeren Frauen (Adaptiert nach [75])

\begin{tabular}{lll}
\hline Endpunkt & RR $(95 \% \mathrm{KI})$ & VE in \% (95\% KI) \\
\hline Laborbestätigte Influenzainfektion & $0,49(0,28-0,85)$ & $51(15-72)$ \\
Influenzaähnliche Erkrankung & $0,96(0,76-1,20)$ & $4(-20-24)$ \\
Mindestens eine lokale UAW & $2,36(1,52-3,66)$ & - \\
Mindestens eine systemische UAW & $1,13(0,74-1,74)$ & - \\
RR relatives Risiko, 95\% KI 95\% Konfidenzintervall, VE Impfeffektivität, UAW unerwünschte Arzneimittelwirkung \\
\hline
\end{tabular}

Personen mit koronarer Herzkrankheit signifikante Effekte nur dann vorlagen, wenn das koronarischämische Ereignis weniger als 1 Jahr zurücklag.

\section{Zusammenfassung und Beurteilung}

Die Daten zeigen, dass die Influenzaimpfung effektiv vor kardiovaskulären Ereignissen schützen kann. Auch wenn laborbestätigte Endpunkte fehlten, wurde die Evidenzqualität insgesamt als hoch bewertet (GRADE- - Tab. 5 ist online als elektronisches Zusatzmaterial beim Bundesgesundheitsblatt unter der Bezeichnung „Anhang zum Hintergrundpapier der STIKO“ abrufbar).

Insbesondere Personen, bei denen ein akutes koronarischämisches Ereignis erst weniger als 1 Jahr zurückliegt, scheinen von der Influenzaimpfung zu profitieren.

\section{Medizinisches Personal}

Als Grundlage für die Beurteilung der Evidenz bei medizinischem Personal diente der systematische Review von Ahmed et al. (AMSTAR-Score 8/11) [70]. In diesen Review gingen 4 ClusterRCTs und 4 Beobachtungsstudien ein, die den Effekt der Influenzaimpfung von medizinischem Personal auf das Auftreten influenzaassoziierter Endpunkte bei betreuten Patienten untersuchten. Die 4 Cluster-RCTs wurden in insgesamt 116 Pflegeeinrichtungen in Schottland $(n=2)$, England und Frankreich durchgeführt. Der Altersdurchschnitt in den Pflegeeinrichtungen betrug 77-86 Jahre. In den Studien wurden die Endpunkte Mortalität jeglicher Ursache, Hospitalisierung jeglicher Ursache, laborbestätigte Influenzaerkrankung und ILI berichtet. Das Verzerrungsrisiko in den RCTs wurde hinsichtlich der Endpunkte Mortalität und Hospitalisierung als niedrig, bezüglich der beiden anderen Endpunkte als hoch bewertet.

In den RCTs konnten die Autoren einen signifikanten Effekt auf die Mortalität (VE: 29\%, 95\% KI: 15-41\%, Evidenzqualität moderat) und ILI bei den betreuten Patienten zeigen (VE: $42 \%$, $95 \% \mathrm{KI}: 26-54 \%$, Evidenzqualität niedrig). Keine signifikanten Effekte wurden gegen laborbestätigte Influenzainfektionen (Evidenzqualität sehr niedrig) oder Hospitalisierungen (Evidenzqualität niedrig) berichtet. Die Herabstufung der Evidenzqualität gemäß GRADE erfolgte, weil einige Endpunkte (Mortalität jeglicher Ursache bzw. Hospitalisierung jeglicher Ursache) nur als Surrogatmarker für Influenzaerkrankungen gelten können und/oder aufgrund eines hohen bzw. sehr hohen Verzerrungsrisikos (bei den Endpunkten laborbestätigte Erkrankung bzw. ILI; GRADE- - Tab. 6 ist online als elektronisches Zusatzmaterial beim Bundesgesundheitsblatt unter der Bezeichnung „Anhang zum Hintergrundpapier der STIKO“ abrufbar).

In den 4 Beobachtungsstudien, die in Frankreich, den Niederlanden, Japan und in den USA durchgeführt wurden, konnte in 3 Studien ein signifikanter Effekt gegen ILI (Evidenzqualität sehr niedrig) und in 2 Studien ein Effekt gegen laborbestätigte Influenzainfektionen nachgewiesen werden. Die Spanne der Effektschätzer war bezüglich beider Endpunkte sehr breit.

\section{Zusammenfassung und Beurteilung} Daten aus RCTs geben Hinweise darauf, dass die Influenzaimpfung von medizinischem Personal die Mortalität und influenzaähnliche Erkrankungen bei älteren Patienten in Pflegeheimen reduzieren kann (Evidenzqualität niedrig bis moderat). Einen Effekt gegen laborbestätigte Erkrankungen bei den betreuten Patienten konnte jedoch nur in den Beobachtungs- studien gezeigt werden, die Evidenzqualität war hierbei sehr niedrig.

\section{Schwangere und deren Neuge- borene}

In der systematischen Literatursuche konnten 4 systematische Reviews identifiziert werden [71-74], die zwischen 2013 und 2014 publiziert worden waren und eine hohe methodische Qualität aufwiesen (AMSTAR-Score 9-11). Während 3 Reviews die Effektivität und Sicherheit bei schwangeren Frauen und deren Kindern untersuchten, fokussierte ein Review [73] auf das Risiko für Frühgeburtlichkeit und fetalen Tod nach Influenzaimpfung. Während einer Aktualisierung der Literatursuche wurde zusätzlich 1 RCT identifiziert [75], der aufgrund seines Veröffentlichungsdatums in keinem der systematischen Reviews enthalten war.

Eine detaillierte Analyse der Studienbasis ergab, dass in dem systematischen Review von McMillan et al. [74] die umfangreichste Datenlage vorlag. Er diente deshalb als Grundlage der vorliegenden Analyse und wurde um den neu identifizierten RCT von Madhi et al. [75] erweitert. Die Re-Analyse der Daten erfolgte stratifiziert nach Endpunkten 1) bei den Müttern sowie 2) bei deren Neugeborenen. Studien zu monovalenten oder adjuvantierten Influenzaimpfstoffen wurden nicht berücksichtigt. Aufgrund der starken Heterogenität der Studien (z.B. verschiedene Populationen, unterschiedliche Definitionen der verwendeten Endpunkte) wurden die Daten nicht statistisch in einer Metaanalyse zusammengefasst.

Bei schwangeren Frauen zeigte der RCT von Madhi et al. [75] einen statistisch signifikanten Effekt gegen laborbestätigte Influenzainfektionen (VE: 51\%, 95\% KI: 15-72\%), nicht jedoch gegen ILI (VE: $4 \%$, 95\% KI: $-20-24 \%)(\bullet$ Tab. 4). Die Evidenzqualität wurde für beide Endpunkte als hoch bewertet (GRADE- $\bullet$ Tab. 7a ist online als elektronisches Zusatzmaterial beim Bundesgesundheitsblatt unter der Bezeichnung „Anhang zum Hintergrundpapier der STIKO“ abrufbar).

Die Dokumentation lokaler und systemischer Nebenwirkungen in diesem RCT 
Tab. 5 Sicherheit der Influenzaimpfung bei schwangeren Frauen. Daten aus 4 Beobachtungsstudien [76-79]

\begin{tabular}{|c|c|c|c|}
\hline Endpunkt & Autor, Jahr & Studiendesign & $\begin{array}{l}\text { Adjustiertes OR } \\
(95 \% \mathrm{KI})\end{array}$ \\
\hline Laborbestätigte Influenzainfektion & Thompson et al. 2014 [79] & Fall-Kontroll & $0,56(0,33-0,95)$ \\
\hline Arztbesuch aufgrund einer influenzaähnlichen Erkrankung & Black et al. 2004 [76] & Kohorte & $1,15(0,98-1,35)$ \\
\hline Schwerwiegende UAW ${ }^{\mathrm{a}}$ & Munoz et al. 2005 [77] & Kohorte & $0,62(0,40-0,98)^{b}$ \\
\hline \multicolumn{4}{|l|}{ Arztbesuch aufgrund neurologischer Erkrankung } \\
\hline - innerhalb von 3 Tagen nach Impfung & Nordin et al. 2013 [78] & Kohorte & $1,12(0,81-1,55)$ \\
\hline - innerhalb von 42 Tagen nach Impfung & Nordin et al. 2013 [78] & Kohorte & $0,92(0,54-1,56)$ \\
\hline Thrombozytopenie innerhalb von 42 Tagen nach Impfung & Nordin et al. 2013 [78] & Kohorte & $0,90(0,68-1,19)$ \\
\hline Präeklampsie & Munoz et al. 2005 [77] & Kohorte & $0,33(0,02-6,01)^{b}$ \\
\hline \multicolumn{4}{|c|}{$\begin{array}{l}\text { OR Odds ratio, } 95 \% \text { KI } 95 \% \text { Konfidenzintervall, UAW unerwünschte Arzneimittelwirkung } \\
\text { aDefiniert als jegliche Hospitalisierung der Mütter } \\
\text { bUnadjustierter Punktschätzer }\end{array}$} \\
\hline
\end{tabular}

Tab. 6 Wirksamkeit und Sicherheit der Influenzaimpfung bei Kindern, bei denen die Mutter die Influenzaimpfung während der Schwangerschaft erhalten hatte. (Adaptiert nach [75, 80])

\begin{tabular}{llll}
\hline Endpunkt & Autor, Jahr & RR (95\% KI) & VE in \% (95\% KI) \\
\hline Laborbestätigte Influenzainfektion & Madhi et al. 2014 [75] & $0,51(0,30-0,88)$ & $49(12-70)$ \\
& Zaman et al. 2008 [80] & $0,37(0,15-0,95)$ & $63(5-85)$ \\
\hline Influenzaähnliche Erkrankung & Madhi et al. 2014 [75] & $1,02(0,94-1,09)-2(-9-6)$ & - \\
Postnataler Tod & Madhi et al. 2014 [75] & $1,19(0,51-2,77)$ & - \\
Frühgeburt (<37 Schwangerschaftswoche) & Madhi et al. 2014 [75] & $1,13(0,85-1,51)$ & - \\
Fehlgeburt ( $\geq 28$ Schwangerschaftswoche) & Madhi et al. 2014 [75] & $1,66(0,72-3,82)$ & - \\
Fehlgeburt (<28 Schwangerschaftswoche) & Madhi et al. 2014 [75] & $0,59(0,14-2,49)$ & - \\
\hline RR relatives Risiko, 95\% KI 95\% Konfidenzintervall, VE Impfeffektivität & & & \\
\hline
\end{tabular}

erfolgte bei geimpften Frauen über einen Zeitraum von einer Woche nach Impfung, schwere Nebenwirkungen wurden über den gesamten Studienzeitraum (bis zur 24. Woche nach der Geburt des Kindes [75]) erhoben. Lokale, nicht jedoch systemische UAWs wurden bei den geimpften Frauen häufiger beobachtet als in der Kontrollgruppe (Evidenzqualität hoch) (• Tab. 4).

In insgesamt 4 Beobachtungsstudien [76-79] fanden sich keine Hinweise auf erhöhte Risiken für schwerwiegende neurologische Ereignisse oder Thrombozytopenien innerhalb von 42 Tagen nach der saisonalen Influenzaimpfung bei den Müttern (•Tab.5). Ebenso fand sich kein erhöhtes Risiko für Präeklampsien. Die Evidenzqualität für diese Endpunkte war sehr niedrig bis niedrig.

Bei Neugeborenen zeigte sich in 2 RCTs $[75,80]$ ein protektiver Effekt gegen laborbestätigte Influenzainfektionen, wenn die Mutter die Influenzaimpfung in der Schwangerschaft erhalten hatte (- Tab. 6). Gegen den unspezifischen
Endpunkt ILI fand sich in der Studie von Madhi et al. [75] kein signifikanter Effekt (VE: -2\%, 95\% KI: -9-6\%). Die Evidenzqualität beider Endpunkte wurde als hoch bewertet (GRADE- - Tab. 7b ist online als elektronisches Zusatzmaterial beim Bundesgesundheitsblatt unter der Bezeichnung „Anhang zum Hintergrundpapier der STIKO“ abrufbar).

Zur Analyse möglicher Nebenwirkungen der Impfung bei Ungeborenen/ Neugeborenen konnte nur einer der zwei RCTs (Madhi et al. [75]) verwendet werden, da nur dieser die Influenzaimpfung mit einer Placebogabe verglich. Hier fanden sich keine Hinweise für eine Häufung von Todesfällen, Frühgeburtlichkeit, Totgeburt oder Fehlgeburt (• Tab. 6). Die Evidenzqualität war moderat bis hoch.

Zusätzlich $\mathrm{zu}$ den oben genannten RCTs wurden Daten aus 12 Beobachtungsstudien [76, 77, 81-90] aus dem Review von McMillan et al. [74] für die Evidenzbewertung herangezogen (• Tab. 7). Auch hier fanden sich keine erhöhten Ri- siken bezüglich der oben beschriebenen Endpunkte. Zudem zeigten sich für kongenitale Malformationen und vermindertes Geburtsgewicht („small for gestational age") kein erhöhtes Risiko (Evidenzqualität sehr niedrig). Einige Beobachtungsstudien fanden einen protektiven Effekt bezüglich Frühgeburtlichkeit (OR zwischen 0,22 [95\% KI: 0,06-0,83] und 0,75 [95\% KI: 0,60-0,94]). In einer Studie war der Effektschätzer hinsichtlich Frühgeburtlichkeit zwar erhöht, statistisch aber nicht signifikant (OR: 2,25, 95 \% KI: 0,88-5,75).

\section{Zusammenfassung und Beurteilung}

Die Daten aus den RCTs zeigen, dass die saisonale Influenzaimpfung sowohl Schwangere als auch deren Neugeborene nach der Geburt effektiv gegen laborbestätigte Influenzaerkrankungen schützt (Evidenzqualität hoch). Die vorliegenden Daten geben bei moderater bis hoher Evidenzqualität keine Hinweise auf ein erhöhtes Risiko von Fehlgeburt, Totgeburt oder Frühgeburt. Ebenso finden sich kei- 
Tab. 7 Wirksamkeit und Sicherheit der Influenzaimpfung bei Kindern, deren schwangere Mütter die Influenzaimpfung erhalten hatten. Daten aus 12 Beobachtungsstudien [76, 77, 81-90] aus dem systematischen Review von Macmillan et al. [74]

\begin{tabular}{|c|c|c|c|c|}
\hline Endpunkt & Autor, Jahr & Studiendesign & $\begin{array}{l}\text { Unadjustiertes OR } \\
(95 \% \mathrm{KI})\end{array}$ & $\begin{array}{l}\text { Adjustiertes OR } \\
(95 \% \mathrm{KI})\end{array}$ \\
\hline \multirow[t]{3}{*}{ Laborbestätigte Influenzainfektion } & Eick et al. 2011 [83] & Kohorten & $0,59(0,37-0,93)$ & - \\
\hline & Benowitz et al. 2010 [81] & Fall-Kontroll & $0,1(0,02-0,4)$ & $0,08(0,02-0,38)$ \\
\hline & Poehling $2011^{\mathrm{a}}$ [89] & Fall-Kontroll & $0,53(0,32-0,88)$ & $0,52(0,30-0,91)$ \\
\hline \multirow[t]{4}{*}{ Influenzaähnliche Erkrankung (ILI) } & Black et al. $2004[76]^{\mathrm{b}}$ & Kohorten & $0,95(-)$ & $0,96(0,89-1,03)$ \\
\hline & Eick et al. 2011 [83] & Kohorten & $0,92(0,73-1,16)$ & - \\
\hline & France et al. 2006 [84] & Kohorten & $0,90(0,80-1,02)$ & $0,96(0,86-1,07)$ \\
\hline & Munoz et al. $2005[77]^{c}$ & Kohorten & $1,19(0,84-1,70)$ & - \\
\hline \multirow[t]{8}{*}{ Frühgeburt ( $<37$ Schwangerschaftswoche) } & Black et al. 2004 [76] & Kohorten & $1,10(0,97-1,26)$ & - \\
\hline & Chambers et al. 2013 [108] & Kohorten & - & $2,25(0,88-5,75)^{\mathrm{d}}$ \\
\hline & Dodds et al. 2012 [82] & Kohorten & $0,84(0,69-1,02)$ & $0,84(0,69-1,02)$ \\
\hline & Legge et al. 2014 [86] & Kohorten & $0,75(0,60-0,93)$ & $0,75(0,60-0,94)$ \\
\hline & Munoz et al. 2005 [77] & Kohorten & $1,42(0,5-4,03)$ & - \\
\hline & Omer et al. 2011 [88] & Kohorten & $0,60(0,41-0,89)$ & $0,54(0,32-0,90)$ \\
\hline & Sheffield et al. 2012 [90] & Kohorten & $0,86(0,78-0,95)$ & - \\
\hline & Louik et al. 2013 [87] & Fall-Kontroll & $0,43(0,13-1,47)$ & $0,22(0,06-0,83)$ \\
\hline Fehlgeburt (>500 g) & Sheffield et al. 2012 [90] & Kohorten & $0,60(0,41-0,86)$ & - \\
\hline Fehlgeburt ohne weitere Angaben & Irving et al. 2013 [85] & Fall-Kontroll & $1,10(-)$ & $1,23(0,53-2,89)$ \\
\hline \multirow[t]{2}{*}{ Kongenitale Malformation } & Munoz et al. 2005 [77] & Kohorten & $0,12(0,01-1,95)$ & - \\
\hline & Sheffield et al. 2012 [90] & Kohorten & $1,01(0,85-1,21)$ & - \\
\hline \multirow[t]{2}{*}{ "Small for gestational age" } & Omer et al. 2011 [88] & Kohorten & $0,74(0,47-1,15)$ & $0,84(0,50-1,40)$ \\
\hline & Sheffield et al. 2012 [90] & Kohorten & $1,00(0,93-1,08)$ & - \\
\hline Postnataler Tod & Sheffield et al. 2012 [90] & Kohorten & $0,55(0,35-0,88)$ & - \\
\hline \multicolumn{5}{|c|}{$\begin{array}{l}\text { OR Odds ratio, } 95 \% \text { KI } 95 \% \text { Konfidenzintervall } \\
\text { aHospitalisierungen mit laborbestätigter Influenzainfektion } \\
\text { 'DArztbesuche mit ILI } \\
\text { cAkute Atemwegsinfektion } \\
\text { dHazard ratio }\end{array}$} \\
\hline
\end{tabular}

ne Hinweise auf Assoziationen zwischen kongenitalen Malformationen bzw. niedrigem Geburtsgewicht und einer Influenzaimpfung während der Schwangerschaft.

\section{Senioren}

Bei der Überarbeitung wurden aus dem systematischen Review von Jefferson et al. (2010, [91]) alle relevanten RCTs analysiert, anschließend wurde die Literatursuche dieses Reviews aktualisiert, um potenziell neue, seither publizierte RCTs zu identifizieren. Zusätzlich erfolgte eine ReAnalyse der in dem systematischen Reviews von Darvishian et al. [92] eingeschlossenen TN-Design Studien. Dieser Studientyp (siehe oben „Impfeffektivität saisonaler Influenzaimpfstoffe") hatte sich gegenüber anderen Beobachtungsstudien als weniger anfällig für systematische Verzerrung gezeigt $[19,93]$ und wurde daher zusätzlich zur Beurteilung der Evidenz herangezogen.

\section{Wirksamkeit der Impfung bei Senioren}

RCTs. 8 der 75 in dem Review von Jefferson et al. eingeschlossenen Studien waren RCTs. Von diesen wurden 4 ausgeschlossen, da entweder ein monovalenter Impfstoff verwendet wurde oder die Vergleichsgruppe ebenfalls eine Intervention (Pneumokokkenimpfung) erhalten hatte. Von den verbleibenden 4 RCTs berichteten 2 Studien Daten zur Wirksamkeit und 3 Studien Daten zur Sicherheit der Influenzaimpfung bei Senioren.

Im RCT von Govaert et al. [65] wurde bei Senioren $(60+)$ aus der Wohnbe- völkerung eine VE von $50 \%$ (95\% KI: 39-65\%) gegen laborbestätigte Influenzainfektionen gemessen (Evidenzqualität hoch, GRADE- $\bullet$ Tab. 8a ist online als elektronisches Zusatzmaterial beim Bundesgesundheitsblatt unter der Bezeichnung „Anhang zum Hintergrundpapier der STIKO“ abrufbar).

Der RCT wurde während der H3N2dominanten Saison 1991/1992 in den Niederlanden durchgeführt und umfasste mehr als 1800 Personen.

Der zweite RCT mit Daten zur Effektivität der Influenzaimpfung bei Senioren wurde während der Saison 1996/97 in Russland durchgeführt [94]. In der von Influenza-A/ H1N1 und Influenza-B dominierten Saison wurde bei Senioren, die in Pflegeinstitutionen lebten, eine nichtsignifikante VE von $49,8 \%$ (95\% KI: -25,5 - 79,9\%) gemes- 


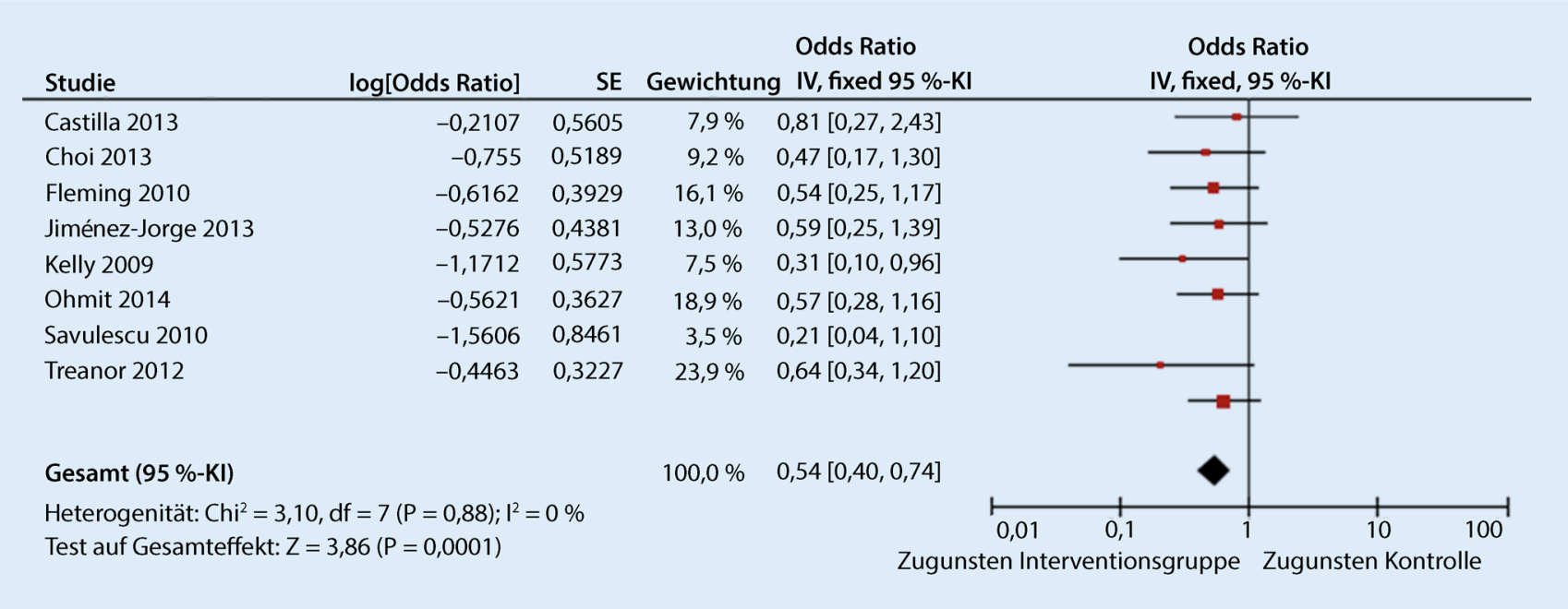

Abb. 5 ム Effektivität der Influenzaimpfung gegen laborbestätigte Influenzainfektionen bei nichthospitalisierten Senioren (65+). Metaanalyse von 9 Originalstudien aus dem Review von Davishian et al. [92], die adjustierte Punktschätzer berichteten. IV Inverse Varianz, KI Konfidenzintervall, $S E$,standard error" (Standardfehler)

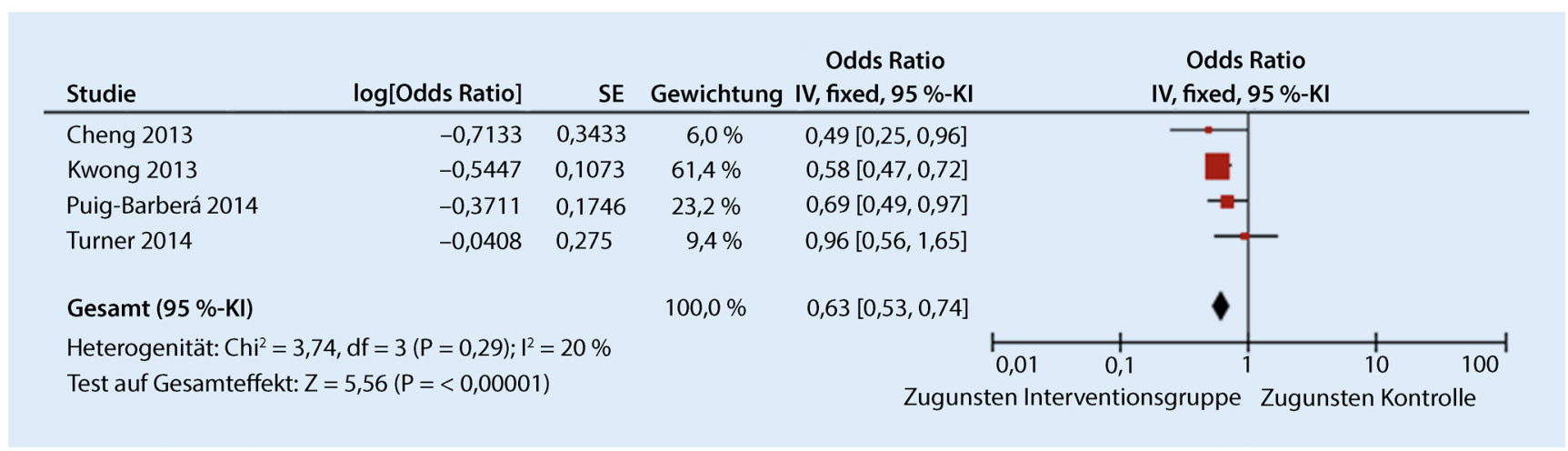

Abb. 6 ॥ Effektivität der Influenzaimpfung gegen laborbestätigte Influenzainfektionen bei hospitalisierten Senioren (65+). Metaanalyse von 4 Original-

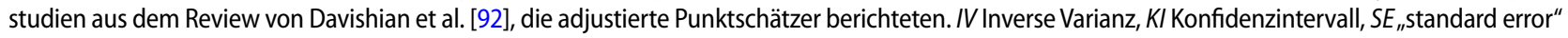
(Standardfehler)

sen. Die Evidenzqualität wurde aufgrund des breiten Konfidenzintervalls (GRADEKriterium „imprecision“) um eine Stufe auf moderat heruntergesetzt (GRADE-• Tab. 8b ist online als elektronisches Zusatzmaterial beim Bundesgesundheitsblatt unter der Bezeichnung „Anhang zum Hintergrundpapier der STIKO“ abrufbar).

Mittels einer systematischen Literatursuche konnten keine weiteren, seit der Publikation des Reviews von Jefferson publizierten RCTs, identifiziert werden.

Beobachtungsstudien. In dem Review von Darvishian et al. [92] wurden 35 Studien aus 15 Ländern eingeschlossen. 25 dieser Studien wurden nach der Pandemie 2009 durchgeführt. In 26 Studien wurden Daten von ambulanten und in 9 Studien
Daten von hospitalisierten Senioren (65+) erhoben. Studien, die ausschließlich Personen mit chronischen Krankheiten eingeschlossen hatten $(n=13)$, wurden von den Autoren nicht analysiert.

Bei 26 Studien beurteilten wir das Verzerrungsrisiko als niedrig, bei 9 Studien (aufgrund fehlender bzw. nicht nachvollziehbarer Adjustierung auf mögliche Confounder) als unklar. Darvishian et al. berichteten die Impfeffektivität stratifiziert 1) nach der Übereinstimmung des Impfstoffes mit den zirkulierenden Viren (gute Übereinstimmung vs. schlechte Übereinstimmung) und 2) nach den WHO-Stadien der geografischen Ausbreitung der Influenza während einer Influenzasaison („sporadische Aktivität“ bis „weitverbreitete Ausbrüche“ [95]).
Während der Influenzasaison konnte eine statistisch signifikante VE von 46$58 \%$ bei guter Übereinstimmung und eine VE von $28-46 \%$ bei schlechter Übereinstimmung mit dem Impfstoff gemessen werden. Da es sich bei diesen Ergebnissen um die gepoolten Daten nichtadjustierter Werte der Originalstudien handelte, führten wir eine zusätzliche Analyse mit den adjustierten Punktschätzern durch, soweit die Daten verfügbar waren:

Die VE gegen laborbestätigte Influenzainfektion lag bei nichthospitalisierten Senioren bei $46 \%$ (95\% KI: 26-60\%, $n=8$ Studien [96-104], - Abb. 5) und bei hospitalisierten Senioren bei 37\% (95\% KI: $26-47 \%, n=4$ Studien [104-107], - Abb. 6). Daten zur Sicherheit der Influ- 
enzaimpfung wurden in dem Review von Darvishian et al. [92] nicht berichtet.

\section{Sicherheit der Influenzaimpfung bei Senioren}

In dem Review von Jefferson et al. [91] wurden Daten zu lokalen und systemischen UAWs berichtet, nicht jedoch zu den von der STIKO definierten Endpunkten (ungeplanter Arztbesuch, Hospitalisierung). In insgesamt 4 RCTs wurden systematische UAWs wie Fieber, Übelkeit und Kopfschmerzen nichtsignifikant häufiger und lokale UAWs (z. B. Schmerzen, Schwellung) signifikant häufiger in der Verumgruppe beobachtet. Die Autoren des Reviews beurteilten das Sicherheitsprofil als akzeptabel.

\section{Zusammenfassung und Beurteilung}

Für Senioren liegt Evidenz von moderater bis hoher Qualität vor, dass die Impfung vor laborbestätigten Influenzainfektionen schützt. Die in randomisierten kontrollierten Studien (RCTs) gemessene Impfeffektivität von $50 \%$ konnte in Beobachtungsstudien bestätigt werden. Das Sicherheitsprofil der Impfung kann als gut bewertet werden.

\section{Zusammenfassung der Evalua- tion}

Die STIKO hat ihre bestehende Impfempfehlung für Indikationsgruppen und für Senioren retrospektiv evaluiert und dabei deutliche Unterschiede in der Qualität der Evidenz in den verschiedenen Personengruppen festgestellt. Die Evidenzqualität - und somit das Vertrauen in die berechnete Impfeffektivität - fiel dabei höher aus, wenn in Studien laborbestätigte Endpunkte berichtet wurden. Unspezifische Endpunkte, wie beispielsweise Hospitalisierungen jeglicher Ursache, führten hingegen i.d.R. zu einer Herabstufung der Evidenzqualität und zu einer größeren Unsicherheit der berechneten Effektivitätsschätzer.

Bei der Bewertung der gefundenen Evidenz muss bedacht werden, dass eine Berechnung der Impfeffektivität nur retrospektiv erfolgen kann und eine Prognose für zukünftige Influenzasaisons nicht möglich ist, weil die Effektivität stark vom jeweils kursierenden Virus abhängt. Die STIKO ist sich bewusst, dass viele der identifizierten Studien nicht in Deutschland durchgeführt wurden und eine Übertragbarkeit der Daten auf die hiesige Situation je nach untersuchtem Parameter unter Umständen eingeschränkt sein könnte.

Die STIKO kommt zu dem Schluss, dass angesichts der hohen influenzabedingten Krankheitslast die Influenzaimpfung auch bei vergleichsweise geringer und saisonal schwankender Impfeffektivität unverändert eine sinnvolle Präventionsmaßnahme darstellt. Die STIKO behält ihre Impfempfehlung für die genannten Indikationsgruppen und für Senioren bei, weist aber darauf hin, dass die evaluierten Indikationsgruppen exemplarischen Charakter haben und keine abschließende Indikationsliste darstellen.

Aufgrund der limitierten Evidenz in einigen Personengruppen betont die STIKO die Notwendigkeit der Durchführung weiterer Studien mit aussagekräftigem Studiendesign und laborbestätigten Endpunkten. Zusätzlich muss die Forschung zur Entwicklung neuer, besserer und breiter wirksamer Impfstoffe vorangetrieben werden.

\section{Korrespondenzadresse}

Cornelius Remschmidt

Fachgebiet Impfprävention

Robert Koch-Institut

Seestraße 10

13353 Berlin, Deutschland

remschmidtc@rki.de

Danksagung. Die Autoren danken Dr. Silke Buda und Dr. Udo Buchholz vom Robert Koch-Institut, Fachgebiet für respiratorisch übertragbare Erkrankungen, für ihre inhaltlich wertvollen Beiträge und für die kritische Durchsicht des Manuskripts.

\section{Einhaltung ethischer Richtlinien}

Interessenkonflikt. C. Remschmidt, T. Harder, O. Wichmann, T. Ledig, S. Wicker und T. Mertens geben an, dass kein Interessenkonflikt besteht. E. Garbe erhielt finanzielle Unterstützung bei der Durchführung von klinischen Studien von GSK und Sanofi Pasteur MSD und erhielt ein Honorar von GSK als Mitglied eines Scientific Advisory Boards. M. Terhardt erhielt Honorare für produktunabhängige Fortbildungsvorträge, die von impfstoffherstellenden Firmen (AstraZeneca, Sanofi Pasteur MSD, GSK) refinanziert wurden. F. Zepp erhielt die Erstattung von Reise- und Übernachtungskosten für produktunabhängige Fortbildungsvorträge die von impfstoffherstellenden Firmen (Sanofi Pasteur MSD, Takeda) refinanziert wurden. Für die Durchführung von klinischen Studien erhielt die Universität Mainz Kostenerstattung.

\section{Literatur}

1. Wu Y, Wu Y, Tefsen B, Shi Y, Gao GF (2014) Bat-derived influenza-like viruses $\mathrm{H} 17 \mathrm{~N} 10$ and H18N11. Trends Microbiol 22(4):183-191

2. Biere B, Bauer B, Schweiger B (2010) Differentiation of influenza $B$ virus lineages Yamagata and Victoria by real-time PCR. J Clin Microbiol 48(4):1425-1427

3. WHO (1980) A revision of the system of nomenclature for influenza viruses: a WHO memorandum. Bull World Health Organ 58(4):585-591

4. Nobusawa E, Sato K (2006) Comparison of the mutation rates of human influenza $A$ and $B$ viruses. J Virol 80(7):3675-3678

5. Kreijtz JH, Fouchier RA, Rimmelzwaan GF (2011) Immune responses to influenza virus infection. Virus Res 162(1-2):19-30

6. Mandell GL, Bennett JE, Dolin R (2010) Mandell, Douglas, and Bennett's principles and practice of infectious diseases, 7. Aufl. Churchill Livingstone, Elsevier, Philadelphia

7. Leung NH, Xu C, Ip DK, Cowling BJ (2015) Review article: the fraction of influenza virus infections that are asymptomatic: a systematic review and meta-analysis. Epidemiology 26(6):862-872

8. Hayward AC, Fragaszy EB, Bermingham A, Wang L, Copas A, Edmunds WJ et al (2014) Comparative community burden and severity of seasonal and pandemic influenza: results of the Flu Watch cohort study. Lancet Respir Med 2(6):445-454

9. Coates BM, Staricha KL, Wiese KM, Ridge KM (2015) Influenza A virus infection, innate immunity, and childhood. JAMA Pediatr 169(10):956-963

10. Mertz D, Kim TH, Johnstone J, Lam PP, Science M, Kuster SP et al (2013) Populations at risk for severe or complicated influenza illness: systematic review and meta-analysis. BMJ 347:55061

11. Thomas RE (2014) Is influenza-like illness a useful concept and an appropriate test of influenza vaccine effectiveness? Vaccine 32(19):2143-2149

12. Monto AS, Gravenstein S, Elliott M, Colopy M, Schweinle J (2000) Clinical signs and symptoms predicting influenza infection. Arch Intern Med 160(21):3243-3247

13. World Health Organization (2014) Influenza (seasonal). Fact sheet No. 211. March 2014. http:// www.who.int/mediacentre/factsheets/fs211/en/ Zugegriffen: 01.11.2016

14. Robert Koch Institute (2014) Bericht zur Epidemiologie der Influenza in Deutschland Saison 2014/15. RKI, Berlin

15. Robert Koch-Institut (2014) Die Arbeitsgemeinschaft Influenza als zentrales Instrument im Gesamtkonzept der Influenzaüberwachung in Deutschland. Epidemiol Bull 40:391-396

16. Grund $\mathrm{S}$, Wichmann $\mathrm{O}$, Mertens $\mathrm{T}$, Hengel $\mathrm{H}$ (2013) Concepts, effectiveness, and perspectives of pandemic and seasonal influenza vaccines. Bundesgesundheitsblatt Gesundheitsforschung Gesundheitsschutz 56(1):76-86

17. Paul-Ehrlich-Institut (2015) Informationen zu den in Deutschland zugelassenen Impfstoffen, den freigegebenen Impfstoffdosen und der Impfstoff-Stammzusammensetzung für die Saison 2015/2016. http://www.pei.de/ $\mathrm{DE} /$ infos/fachkreise/impfungen-impfstoffe/ influenza-grippeimpfstoffe-saisonal/ influenza-grippeimpfstoffe-node.html. Zugegriffen: 03. Feb 2016

18. Orenstein WA, Bernier RH, Hinman AR (1988) Assessing vaccine efficacy in the field. Further observations. Epidemiol Rev 10:212-241 
19. De Serres G, Skowronski DM, Wu XW, Ambrose CS (2013) The test-negative design: validity, accuracy and precision of vaccine efficacy estimates compared to the gold standard of randomised placebo-controlled clinical trials. Euro Surveill. doi:10.2807/1560-7917.es2013.18.37.20585

20. Jackson ML, Nelson JC (2013) The test-negative design for estimating influenza vaccine effectiveness. Vaccine 31(17):2165-2168

21. Skowronski DM, Janjua NZ, De Serres G (2013) Understanding suboptimal influenza vaccine effectiveness within the agent, host, and environment paradigm. Clin Infect Dis 57(3):476-477

22. Beyer WE, de Bruijn IA, Palache AM, Westendorp RG, Osterhaus AD (1999) Protection against influenza after annually repeated vaccination: a meta-analysis of serologic and field studies. Arch Intern Med 159(2):182-188

23. Ohmit SE, Petrie JG, Malosh RE, Cowling BJ, Thompson MG, Shay DK et al (2013) Influenza vaccine effectiveness in the community and the household. Clin Infect Dis 56(10):1363-1369

24. Ständige Impfkommission (STIKO) (2015) Mitteilung der Ständigen Impfkommission am Robert Koch-Institut (RKI). Empfehlungen der Ständigen Impfkommission (STIKO) am Robert Koch-Institut. Robert Koch-Institut, Berlin, Deutschland

25. Ständige Impfkommission (STIKO) (2014) Standardvorgehensweise (SOP) der Ständigen Impfkommission (STIKO) für die systematische Entwicklung von Impfempfehlungen. Version 2.0 vom 06.02.2014. http://www.rki.de/DE/Content/ Kommissionen/STIKO/Aufgaben_Methoden/ SOP.pdf?_blob=publicationFile Zugegriffen: 01.11 .2016

26. Shea BJ, Grimshaw JM, Wells GA, Boers $M$, Andersson N, Hamel C et al (2007) Development of AMSTAR: a measurement tool to assess the methodological quality of systematic reviews. Bmc Med Res Methodol 7:10

27. Moher D, Liberati A, Tetzlaff J, Altman DG, Group $P$ (2009) Preferred reporting items for systematic reviews and meta-analyses: the PRISMA statement. Ann Intern Med 151(4):264-269 (W64)

28. Higgins J, Green S (2011) Cochrane Handbook for Systematic Reviews of Interventions Version 5.1.0. The Cochrane Collaboration, London, England. http://handbook.cochrane.org

29. Critical Appraisal Skills Programme (CASP) (2014) CASP checklists. CASP, Oxford

30. Guyatt GH, Oxman AD, Schunemann HJ, Tugwell P, Knottnerus A (2011) GRADE guidelines: a new series of articles in the Journal of Clinical Epidemiology. J Clin Epidemiol 64(4):380-382

31. Guyatt $G H$, Oxman AD, Vist GE, Kunz R, FalckYtter Y, Alonso-Coello P et al (2008) GRADE: an emerging consensus on rating quality of evidence and strength of recommendations. BMJ 336(7650):924-926

32. Guyatt GH, Thorlund $K$, Oxman AD, Walter SD, Patrick D, Furukawa TA et al (2013) GRADE guidelines: 13. Preparing summary of findings tables and evidence profiles-continuous outcomes. J Clin Epidemiol 66(2):173-183

33. Van Kerkhove MD, Vandemaele KA, Shinde V, Jaramillo-Gutierrez G, Koukounari A, Donnelly CA et al (2011) Risk factors for severe outcomes following 2009 influenza A (H1N1) infection: a global pooled analysis. PLOS Med 8(7):e1001053

34. Buda S, Kopke K, Haas W (2010) Epidemiological characteristics of the influenza pandemic (H1N1) 2009 in Germany based on the mandatory notification of cases. Bundesgesundheitsblatt
Gesundheitsforschung Gesundheitsschutz 53(12):1223-1230

35. Remschmidt C, Wichmann O, Harder T (2014) Methodological quality of systematic reviews on influenza vaccination. Vaccine 32(15):1678-1684

36. Atashili J, Kalilani L, Adimora AA (2006) Efficacy and clinical effectiveness of influenza vaccines in HIV-infected individuals: a meta-analysis. BMC Infect Dis 6:138

37. Remschmidt C, Wichmann O, Harder T (2014) Influenza vaccination in HIV-infected individuals: systematic review and assessment of quality of evidence related to vaccine efficacy, effectiveness and safety. Vaccine 32(43):5585-5592

38. Madhi SA, Maskew M, Koen A, Kuwanda L, Besselaar TG, Naidoo D et al (2011) Trivalent inactivated influenza vaccine in African adults infected with human immunodeficient virus: double blind, randomized clinical trial of efficacy, immunogenicity, and safety. Clin Infect Dis 52(1):128-137

39. Tasker SA, Treanor JJ, Paxton WB, Wallace MR (1999) Efficacy of influenza vaccination in HIV-infected persons. A randomized, doubleblind, placebo-controlled trial. Ann Intern Med 131(6):430-433

40. Ranieri R, Veronelli A, Santambrogio C, Pontiroli $A E$ (2005) Impact of influenza vaccine on response to vaccination with pneumococcal vaccine in HIV patients. AIDS Res Hum Retroviruses 21(5):407-409

41. Yamanaka H, Teruya K, Tanaka M, Kikuchi Y, Takahashi T, Kimura S et al (2005) Efficacy and immunologic responses to influenza vaccine in HIV-1-infected patients. J Acquir Immune Defic Syndr 39(2):167-173

42. Fine $A D$, Bridges $C B$, De Guzman $A M$, Glover $L$, Zeller B, Wong SJ et al (2001) Influenza A among patients with human immunodeficiency virus: an outbreak of infection at a residential facility in New York City. Clin Infect Dis 32(12):1784-1791

43. Madhi SA, Dittmer S, Kuwanda L, Venter M, Cassim H, Lazarus E et al (2013) Efficacy and immunogenicity of influenza vaccine in HIVinfected children: a randomized, double-blind, placebo controlled trial. AIDS 27(3):369-379

44. Remschmidt C, Wichmann O, Harder T (2015) Vaccines for the prevention of seasonal influenza in patients with diabetes: systematic review and meta-analysis. BMC Med 13(1):53

45. Hak E, Nordin J, Wei F, Mullooly J, Poblete S, Strikas R et al (2002) Influence of high-risk medical conditions on the effectiveness of influenza vaccination among elderly members of 3 large managed-care organizations. Clin Infect Dis 35(4):370-377

46. Heymann AD, Shapiro Y, Chodick G, Shalev V, Kokia E, Kramer E et al (2004) Reduced hospitalizations and death associated with influenza vaccination among patients with and without diabetes. Diabetes Care 27(11):2581-2584

47. Isotani $H(2000)$ Use of influenza vaccine in patients with diabetes, particularly those with complications of cardiac or renal disease. Intern Med 39(10):862

48. Rodriguez-Blanco T, Vila-Corcoles A, de Diego C, Ochoa-Gondar O, Valdivieso E, Bobe F et al (2012) Relationship between annual influenza vaccination and winter mortality in diabetic people over 65 years. Hum Vaccin Immunother 8(3):363-370

49. Schade CP, McCombs MA (2000) Influenza immunization and mortality among diabetic medicare beneficiaries in West Virginia. W V Med J 96(3):444-448
50. Selvais PL, Hermans MP, Donckier JE, Buysschaert M (1997) Reported rates, incentives, and effectiveness of major vaccinations in 501 attendees at two diabetes clinics. Diabetes Care 20(7):1212-1213

51. The American Lung Association Asthma Clinical Research Centers (2001) The safety of inactivated influenza vaccine in adults and children with asthma. N Engl J Med 345(21):1529-1536

52. Colquhoun AJ, Nicholson KG, Botha JL, Raymond NT (1997) Effectiveness of influenza vaccine in reducing hospital admissions in people with diabetes. Epidemiol Infect 119(3):335-341

53. Gasparini R, Amicizia D, Lai PL, Rossi S, Panatto D (2013) Effectiveness of adjuvanted seasonal influenza vaccines (Inflexal V (R) and Fluad (R)) in preventing hospitalization for influenza and pneumonia in the elderly: a matched case-control study. Hum Vaccin Immunother 9(1):144-152

54. Lau D, Eurich DT, Majumdar SR, Katz A, Johnson JA (2013) Effectiveness of influenza vaccination in working-age adults with diabetes: a populationbased cohort study. Thorax 68(7):658-663

55. Looijmans-Van den Akker I, Verheij TJ, Buskens E, Nichol KL, Rutten GE, Hak E (2006) Clinical effectiveness of first and repeat influenza vaccination in adult and elderly diabetic patients. Diabetes Care 29(8):1771-1776

56. Wang IK, Lin CL, Chang YC, Lin PC, Liang CC, Liu YL et al (2013) Effectiveness of influenza vaccination in elderly diabetic patients: a retrospective cohort study. Vaccine 31(4):718-724

57. Remschmidt C, Wichmann O, Harder T (2014) Influenza vaccination in patients with end-stage renal disease: systematic review and assessment of quality of evidence related to vaccine efficacy, effectiveness, and safety. BMC Med 12(1):244

58. Bond TC, Spaulding AC, Krisher J, McClellan W (2012) Mortality of dialysis patients according to influenza and pneumococcal vaccination status. Am J Kidney Dis 60(6):959-965

59. Gilbertson DT, Unruh M, McBean AM, Kausz AT, Snyder JJ, Collins AJ (2003) Influenza vaccine delivery and effectiveness in end-stage renal disease. Kidney Int 63(2):738-743

60. McGrath LJ, Kshirsagar AV, Cole SR, Wang L, Weber DJ, Sturmer T et al (2012) Influenza vaccine effectiveness in patients on hemodialysis: an analysis of a natural experiment. Arch Intern Med 172(7):548-554

61. Slinin Y, Foley RN, Collins AJ (2006) Clinical epidemiology of pneumonia in hemodialysis patients: the USRDS waves 1, 3, and 4 study. Kidney Int 70(6):1135-1141

62. Wang IK, Lin CL, Lin PC, Liang CC, Liu YL, Chang CT et al (2013) Effectiveness of influenza vaccination in patients with end-stage renal disease receiving hemodialysis: a population-based study. PLOS ONE 8(3):e58317

63. Cates CJ, Rowe BH (2013) Vaccines for preventing influenza in people with asthma. Cochrane Database Syst Rev 2:CD000364. doi:10.1002/14651858.cd000364.pub4

64. Bueving HJ, Bernsen RM, de Jongste JC, van Suijlekom-Smit LW, Rimmelzwaan GF, Osterhaus AD et al (2004) Influenza vaccination in children with asthma: randomized double-blind placebocontrolled trial. Am J Respir Crit Care Med 169(4):488-493

65. Govaert TM, Thijs CT, Masurel N, Sprenger MJ, Dinant GJ, Knottnerus JA (1994) The efficacy of influenza vaccination in elderly individuals. 
A randomized double-blind placebo-controlled trial. JAMA 272(21):1661-1665

66. Kmiecik T, Arnoux S, Kobryn A, Gorski P (2007) Influenza vaccination in adults with asthma: safety of an inactivated trivalent influenza vaccine. J Asthma 44(10):817-822

67. Nicholson KG, Nguyen-Van-Tam JS, Ahmed AH, Wiselka MJ, Leese J, Ayres J et al (1998) Randomised placebo-controlled crossover trial on effect of inactivated influenza vaccine on pulmonary function in asthma. Lancet 351(9099):326-331

68. Udell JA, Zawi R, Bhatt DL, Keshtkar-Jahromi M, Gaughran F, Phrommintikul A et al (2013) Association between influenza vaccination and cardiovascular outcomes in high-risk patients: a meta-analysis. JAMA 310(16):1711-1720

69. De Villiers PJ, Steele AD, Hiemstra LA, Rappaport R, Dunning AJ, Gruber WC et al (2009) Efficacy and safety of a live attenuated influenza vaccine in adults 60 years of age and older. Vaccine 28(1):228-234

70. Ahmed F, Lindley MC, Allred N, Weinbaum CM, Grohskopf L (2014) Effect of influenza vaccination of healthcare personnel on morbidity and mortality among patients: systematic review and grading of evidence. Clin Infect Dis 58(1):50-57

71. Jefferson T, Di Pietrantonj C, Rivetti A, Bawazeer GA, Al-Ansary LA, Ferroni E (2014) Vaccines for preventing influenza in healthy adults. Cochrane Database Syst Rev 3:CD001269

72. Galvao TF, Silva MT, Zimmermann IR, Lopes LA, Bernardo EF, Pereira MG (2013) Influenza vaccination in pregnant women: a systematic review. Isrn Prev Med 2013:879493

73. Fell DB, Platt RW, Lanes A, Wilson K, Kaufman JS, Basso $O$ et al (2015) Fetal death and preterm birth associated with maternal influenza vaccination: systematic review. BJOG 122(1):17-26

74. McMillan M, Kralik D, Porritt K, Marshall H (2014) Influenza vaccination during pregnancy: a systematic review of effectiveness and adverse events. JBI Database Syst Rev Implement Rep 12(6):2014

75. Madhi SA, Cutland CL, Kuwanda L, Weinberg A, Hugo A, Jones S et al (2014) Influenza vaccination of pregnant women and protection of their infants. N Engl J Med 371(10):918-931

76. Black SB, Shinefield HR, France EK, Fireman BH, Platt ST, Shay D et al (2004) Effectiveness of influenza vaccine during pregnancy in preventing hospitalizations and outpatient visits for respiratory illness in pregnant women and their infants. Am J Perinatol 21(6):333-339

77. Munoz FM, Greisinger AJ, Wehmanen OA, Mouzoon ME, Hoyle JC, Smith FA et al (2005) Safety of influenza vaccination during pregnancy. Am J Obstet Gynecol 192(4):1098-1106

78. Nordin JD, Kharbanda EO, Benitez GV, Nichol K, Lipkind H, Naleway A et al (2013) Maternal safety of trivalent inactivated influenza vaccine in pregnant women. Obstet Gynecol 121(3):519-525

79. Thompson MG, Li DK, Shifflett P, Sokolow LZ, Ferber JR, Kurosky S et al (2014) Effectiveness of seasonal trivalent influenza vaccine for preventing influenza virus illness among pregnant women: a population-based case-control study during the 2010-2011 and 2011-2012 influenza seasons. Clin Infect Dis 58(4):449-457

80. Zaman K, Roy E, Arifeen SE, Rahman M, Raqib R, Wilson E et al (2008) Effectiveness of maternal influenza immunization in mothers and infants. N Engl J Med 359(15):1555-1564

81. Benowitz I, Esposito DB, Gracey KD, Shapiro ED, Vazquez M (2010) Influenza vaccine given to pregnant women reduces hospitalization due to influenza in their infants. Clin Infect Dis 51(12):1355-1361

82. Dodds L, Macdonald N, Scott J, Spencer A, Allen VM, McNeil S (2012) The association between influenza vaccine in pregnancy and adverse neonatal outcomes. J Obstet Gynaecol Can 34(8):714-720

83. Eick AA, Uyeki TM, Klimov A, Hall H, Reid R, Santosham M et al (2011) Maternal influenza vaccination and effect on influenza virus infection in young infants. Arch Pediatr Adolesc Med 165(2):104-111

84. France EK, Smith-Ray R, McClure D, Hambidge S, Xu S, Yamasaki K et al (2006) Impact of maternal influenza vaccination during pregnancy on the incidence of acute respiratory illness visits among infants. Arch Pediatr Adolesc Med 160(12):12771283

85. Irving SA, Kieke BA, Donahue JG, Mascola MA, Baggs J, DeStefano F et al (2013) Trivalent inactivated influenza vaccine and spontaneous abortion. Obstet Gynecol 121(1):159-165

86. Legge A, Dodds L, MacDonald NE, Scott J, McNeil $S$ (2014) Rates and determinants of seasonal influenza vaccination in pregnancy and association with neonatal outcomes. CMAJ 186(4):E157-E164

87. Louik C, Ahrens K, Kerr S, Pyo J, Chambers C, Jones KL et al (2013) Risks and safety of pandemic H1N1 influenza vaccine in pregnancy: exposure prevalence, preterm delivery, and specific birth defects. Vaccine 31(44):5033-5040

88. Omer SB, Goodman D, Steinhoff MC, Rochat R, Klugman KP, Stoll BJ et al (2011) Maternal influenza immunization and reduced likelihood of prematurity and small for gestational age births: a retrospective cohort study. PLOS Med 8(5):e1000441

89. Poehling KA, Szilagyi PG, Staat MA, Snively BM Payne DC, Bridges CB et al (2011) Impact of maternal immunization on influenza hospitalizations in infants. Am J Obstet Gynecol 204(6 Suppl 1):141-148

90. Sheffield JS, Greer LG, Rogers VL, Roberts SW, Lytle H, McIntire DD et al (2012) Effect of influenza vaccination in the first trimester of pregnancy. Obstet Gynecol 120(3):532-537

91. Jefferson T, Di Pietrantonj C, Al-Ansary LA, Ferroni E, Thorning S, Thomas RE (2010) Vaccines for preventing influenza in the elderly. Cochrane Database Syst Rev 2:CD004876

92. Darvishian M, Bijlsma MJ, Hak E, van den Heuvel ER (2014) Effectiveness of seasonal influenza vaccine in community-dwelling elderly people: a meta-analysis of test-negative design case-control studies. Lancet Infect Dis 14(12):1228-1239

93. Remschmidt C, Wichmann O, Harder T (2015) Frequency and impact of confounding by indication and healthy vaccinee bias in observational studies assessing influenza vaccine effectiveness: a systematic review. BMC Infect Dis 15:429

94. Rudenko LG, Arden NH, Grigorieva E, Naychin A, Rekstin A, Klimov Al et al (2000) Immunogenicity and efficacy of Russian live attenuated and US inactivated influenza vaccines used alone and in combination in nursing home residents. Vaccine 19(2-3):308-318

95. European Center for Disease Prevention and Control. Indicators of influenza activity. http:// ecdc.europa.eu/en/healthtopics/influenza/ surveillance/Pages/indicators_influenza.aspx. Zugegriffen: 27.01.2016.
96. Castilla J, Martinez-Baz I, Martinez-Artola V, Reina G, Pozo F, Garcia Cenoz M et al (2013) Decline in influenza vaccine effectiveness with time after vaccination, Navarre, Spain, season 2011/12. Euro Surveill 18:5

97. Choi WS, Noh JY, Seo YB, Baek JH, Lee J, Song JY et al (2013) Case-control study of the effectiveness of the 2010-2011 seasonal influenza vaccine for prevention of laboratory-confirmed influenza virus infection in the Korean adult population. Clin Vaccine Immunol 20(6):877-881

98. Fleming DM, Andrews NJ, Ellis JS, Bermingham A, Sebastianpillai P, Elliot AJ et al (2010) Estimating influenza vaccine effectiveness using routinely collected laboratory data. J Epidemiol Community Health 64(12):1062-1067

99. Jimenez-Jorge S, de Mateo S, Delgado-Sanz C, Pozo F, Casas I, Garcia-Cenoz M et al (2013) Effectiveness of influenza vaccine against laboratoryconfirmed influenza, in the late 2011-2012 season in Spain, among population targeted for vaccination. BMC Infect Dis 13:441

100. Kelly H, Carville K, Grant K, Jacoby P, Tran T, Barr I (2009) Estimation of influenza vaccine effectiveness from routine surveillance data. PLOS ONE 4(3):e5079

101. Ohmit SE, Thompson MG, Petrie JG, Thaker SN, Jackson ML, Belongia EA et al (2014) Influenza vaccine effectiveness in the 2011-2012 season: protection against each circulating virus and the effect of prior vaccination on estimates. Clin Infect Dis 58(3):319-327

102. Pebody RG, Andrews N, Fleming DM, McMenamin J, Cottrell S, Smyth B et al (2013) Age-specific vaccine effectiveness of seasonal 2010/2011 and pandemic influenza $A(H 1 N 1) 2009$ vaccines in preventing influenza in the United Kingdom. Epidemiol Infect 141(3):620-630

103. Savulescu C, Valenciano M, de Mateo S, Larrauri A (2010) Estimating the influenza vaccine effectiveness in elderly on a yearly basis using the Spanish influenza surveillance network-pilot case-control studies using different control groups, 2008-2009 season, Spain. Vaccine 28(16):2903-2907

104. Treanor JJ, Talbot HK, Ohmit SE, Coleman LA, Thompson MG, Cheng PY et al (2012) Effectiveness of seasonal influenza vaccines in the United States during a season with circulation of all three vaccine strains. Clin Infect Dis 55(7):951-959

105. Cheng AC, Holmes M, Irving LB, Brown SG, Waterer GW, Korman TM et al (2013) Influenza vaccine effectiveness against hospitalisation with confirmed influenza in the $2010-11$ seasons: a test-negative observational study. PLOS ONE 8(7):e68760

106. Kwong JC, Campitelli MA, Gubbay JB, Peci A, Winter AL, Olsha R et al (2013) accine effectiveness against laboratory-confirmed influenza hospitalizations among elderly adults during the 2010-2011 season. Clin Infect Dis 57(6):820-827

107. Puig-Barbera J, Natividad-Sancho A, Launay O, Burtseva E, Ciblak MA, Tormos A et al (2014) 2012-2013 Seasonal influenza vaccine effectiveness against influenza hospitalizations: results from the global influenza hospital surveillance network. PLOS ONE 9(6):e100497

108. Chambers et al. (2013) Risks and safety of pandemic $\mathrm{H} 1 \mathrm{~N} 1$ influenza vaccine in pregnancy: birth defects, spontaneous abortion, preterm delivery, and small for gestational age infants. Vaccine 31(44):5026-5032. doi: 10.1016/j.vaccine.2013.08.097 